\title{
On a Class of Linear Cooperative Systems with Spatio-temporal Degenerate Potentials
}

\author{
P. Álvarez-Caudevilla@, F. Belinchón and C. Brändle
}

\begin{abstract}
This paper analyses a class of parabolic linear cooperative systems in a cylindrical domain with degenerate spatio-temporal potentials. In other words, potentials vanish in some non-empty connected subdomains which are disjoint and increase in size temporally. Then, the vanishing subdomains for the potentials are not cylindrical. Following a similar idea to the semiclassical analysis behaviour, but done here for parabolic problems, under these geometrical assumptions, the asymptotic behaviour of the system is ascertained when a parameter, in front of these potentials, goes to infinity. In particular, the strong convergence of the solutions of the system is obtained using energy methods and the theory associated with the $\Gamma$-convergence. Also, the exponential decay of the solutions to zero in the exterior of the subdomains where the potentials vanish is achieved.
\end{abstract}

Mathematics Subject Classification. 35A15, 35K45.

Keywords. Eigenvalue problems, asymptotic analysis, degenerate potentials, spatio-temporal coefficients.

\section{Introduction}

The analysis shown in this paper focuses on the asymptotic behaviour, when the parameter $\lambda>0$ goes to infinity, of solutions $\mathbf{U}_{\lambda}=\left(u_{1, \lambda}, u_{2, \lambda}\right)^{T}$ to a parabolic system defined on the cylindrical domain with spatio-temporal coefficients $Q_{T}:=\Omega \times(0, T)$, where $\Omega \subset \mathbb{R}^{N}$ is an open set and $N \geq 1$, $T>0$, and with spatio-temporal coefficients. In particular, we consider the linear matrix problem

$$
\begin{cases}\partial_{t} \mathbf{U}_{\lambda}+\left(\mathcal{L}+\mathcal{P}_{\lambda}\right) \mathbf{U}_{\lambda}=\mathbf{F}_{\lambda} & \text { in } Q_{T}, \\ \mathbf{U}=0 & \text { in } \partial \Omega \times(0, T), \\ \mathbf{U}_{\lambda}(x, 0)=\mathbf{U}_{0, \lambda}(x) & \text { in } \Omega,\end{cases}
$$

This paper has been partially supported by the Ministry of Science and Innovation of Spain under research project PID2019-106122GB-I00. 


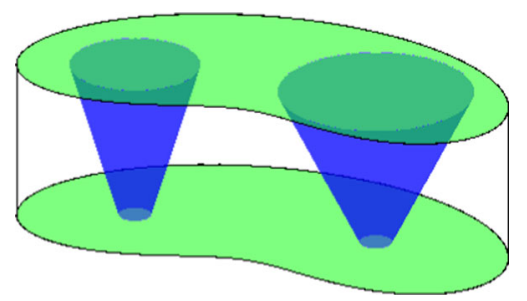

Figure 1. Representation of domain $\Omega \times(0, T)$. The cones correspond to the sets $O_{a_{i}}$

where the vector $\mathbf{U}_{0, \lambda}=\left(u_{01, \lambda}, u_{02, \lambda}\right)^{T} \in \mathbf{H}_{0}^{1}(\Omega):=H_{0}^{1}(\Omega) \times H_{0}^{1}(\Omega)$ represents the initial condition of our parabolic problem, $\mathbf{F}_{\lambda}:=\left(f_{1, \lambda}, f_{2, \lambda}\right)^{T} \in$ $\mathbf{L}^{2}\left(Q_{T}\right):=L^{2}\left(0, T, L^{2}(\Omega)\right) \times L^{2}\left(0, T, L^{2}(\Omega)\right)$ and

$$
\mathcal{L}+\mathcal{P}_{\lambda}:=\left(\begin{array}{cc}
-\Delta & 0 \\
0 & -\Delta
\end{array}\right)+\left(\begin{array}{cc}
\lambda a_{1}(x, t) & -\alpha_{1}(x, t) \\
-\alpha_{2}(x, t) & \lambda a_{2}(x, t)
\end{array}\right) .
$$

Moreover, the coefficients considered in the problem are spatially and temporally heterogeneous in the sense that

$$
a_{i}: Q_{T} \rightarrow \mathbb{R}^{+} \cup\{0\}, \quad \alpha_{i}: Q_{T} \rightarrow \mathbb{R}^{+}, \quad i=1,2
$$

are bounded measurable functions, with $a_{i}$ non-negative and $\alpha_{i}$ positive, and such that the domains

$$
O_{a_{i}}:=\operatorname{Int}\left(\left\{(x, t) \in Q_{T}: a_{i}(x, t)=0\right\}\right) \neq \emptyset,
$$

are connected sets with boundary of zero Lebesgue measure in $\mathbb{R}^{N}$. Furthermore, from a structural point of view, we suppose that these two regions do not intersect, for any time $t \in(0, T)$. In other words, there exist two cylinders $C_{1}, C_{2} \in Q_{T}$ such that $O_{a_{1}} \subset C_{1}, O_{a_{2}} \subset C_{2}$ and such that $C_{1}$ and $C_{2}$ satisfy $C_{1} \cap C_{2}=\emptyset$ for every $t \in(0, T)$; see Fig. 1 .

Note that we have not considered any pathological sets due to the fact that we are interested in seeing how the domains evolve geometrically under the conditions of our potentials.

As it will be shown throughout this work, the asymptotic behaviour of $\mathbf{U}_{\lambda}$ strongly depends on the nature of potentials $a_{i}$. As a particular example, if the problem $\left(P_{\lambda}\right)$ were an eigenvalue problem we might face two different situations: either there exist non-empty regions where $a_{i}$ vanish. So that, due to the monotonicity of the principal eigenvalue with respect to the domain (see $[4,6,14]$ for further details), the first eigenvalue of $\left(P_{\lambda}\right)$ is bounded above and the limit problem has a bounded principal eigenvalue associated with an eigenfunction which concentrates on these regions. Or the first eigenvalue diverges when $\lambda \rightarrow \infty$ if the potentials are strictly positive with the associated eigenfunction being positive everywhere inside the cylindrical domain. Therefore, we will focus on analysing the situations where we have degenerate spatial and temporal potentials $a_{i}$, as described above. 


\subsection{Organization of the Paper and Main Results}

In Sect. 2, we deal with the stationary version of $\left(P_{\lambda}\right)$, avoiding any dependence on time. For that particular problem, Lax-Milgram theorem shows the existence and uniqueness of weak solutions $\mathbf{U}_{\lambda}$. Then, a standard application of the $\Gamma$-convergence theory based on energy functionals proves the strong convergence of $\mathbf{U}_{\lambda}$ when $\lambda \rightarrow \infty$ to the solution $\mathbf{U} \in H_{0}^{1}\left(K_{a_{1}}\right) \times H_{0}^{1}\left(K_{a_{2}}\right)$ of a limit elliptic problem (see $\left(P_{\infty}^{s}\right)$ ), with $K_{a_{i}}$ being the regions where the potentials $a_{i}$ vanish at any fixed time $t$. This result is proved in Proposition 2.3 and based on results shown in [3]. Note that we have used the same notation for the solution of the stationary problem and the parabolic problem $\left(P_{\lambda}\right)$, even though they are different. However, since the stationary problem will be used just as an auxiliary problem, we prefer to avoid any extra notation, irrelevant for the final purpose of the paper.

The existence and regularity of solutions to $\left(P_{\lambda}\right)$ is stated in Sect. 3. Note that, since $\left(P_{\lambda}\right)$ is a classical system of parabolic equations, existence, uniqueness, and regularity of the weak solutions follow from the standard Galerkin method. In particular, under our hypothesis about the initial condition, we obtain that the solution $\mathbf{U}_{\lambda} \in \mathbf{H}_{0}^{1}\left(Q_{T}\right):=L^{2}\left(0, T, H_{0}^{1}(\Omega)\right) \times L^{2}\left(0, T, H_{0}^{1}(\Omega)\right)$ is continuous in time and $\left(P_{\lambda}\right)$ is satisfied in a weak sense.

Finally, as we have said, the main goal of this paper is the study of the behaviour of the solution of problem $\left(P_{\lambda}\right), \mathbf{U}_{\lambda}$, when the parameter $\lambda$ tends to infinity. To this aim, in Sect. 5, we first obtain an estimation for $\mathbf{U}_{\lambda}$

$$
\sup _{\lambda}\left(\lambda \int_{Q_{T}}\left(\mathcal{A} \mathbf{U}_{\lambda}\right) \cdot \mathbf{U}_{\lambda} \mathrm{d} x \mathrm{~d} t\right) \leq C, \quad \text { for } C \text { a positive constant, }
$$

which implies that, at the limit, the components $u_{i}$ must be equal to zero almost everywhere, from now on a.e., in any set of the form $\left\{a_{i}>\varepsilon\right\}$ with $\varepsilon>0$. Indeed, considering the union for $n \in \mathbb{N}$ of the sets with $\varepsilon=1 / n$, we actually obtain that $u_{i}=0$ a.e in $Q_{T} \backslash O_{a_{i}}$, where the vanishing subdomains $O_{a_{i}}$ are defined in (1.2). Note, even though we are assuming a periodic-parabolic problem, the results obtained in this work follow an analogous asymptotic behaviour if considering an elliptic problem with heterogeneous potentials. Those similar elliptic cooperative systems were analysed previously in $[1,3]$.

The main result proved in see Sect. 5 shows that $\mathbf{U}_{\lambda}$ converges strongly in $\mathbf{H}_{0}^{1}\left(Q_{T}\right)$ to the unique (weak) solution $\mathbf{U}$ of the limit problem

$$
\begin{cases}\partial_{t} \mathbf{U}+\mathcal{L} \mathbf{U}=\mathbf{F} & \text { in } Q_{T}, \\ \mathbf{U}(x, 0)=\mathbf{U}_{0}(x) & \text { in } \Omega,\end{cases}
$$

with $u_{i}=0$ a.e. in $Q_{T} \backslash O_{a_{i}}$. The uniqueness of $\mathbf{U}$ is proved in Sect. 4 . The convergence of weak solutions of $\left(P_{\lambda}\right)$ was already observed in [13] in a single equation setting, as a starting point for a more detailed analysis about the associated semigroup. This was then used in [13] to analyse the asymptotic behaviour of a non-linear periodic-parabolic problem of logistic type (first analysed by Hess [16]; see also [14]) $\partial_{t} u-\Delta u=\mu u-a(x, t) u^{p}$. This equation is used in some models of population dynamics.

Observe that (1.4) is not a standard Cauchy-Dirichlet problem for the heat equation, since each component of the solution $\mathbf{U}$ is defined just in 
$O_{a_{i}}$ and the sets $O_{a_{i}}$ can be in general non-cylindrical domains; see Fig. 1. These types of problems with non-cylindrical domains appear in various applications and have been under analysis in other contexts for related problems to (1.4); see for example [9-12,14,19] and the references therein. In this work, we show the existence and uniqueness of its solution. Indeed, in Sect. 4, a simple energy bound proves that the solution $\mathbf{U}$ of $\left(P_{\infty}\right)$ has to be unique if it exists. To prove this result, we use the energy methods performed in Sect. 2 for the stationary-associated problem. These calculations can be seen as the continuation of a previous work, [3], where the stationary problem was addressed through the $\Gamma$-convergence theory and the paper [2], using different methodologies.

Furthermore, we should mention that in the particular situation when

$$
f_{\lambda}:=f_{1, \lambda}=f_{2, \lambda} \quad a:=a_{1}=a_{2} \quad \alpha:=\alpha_{1}=\alpha_{2} \quad w_{0, \lambda}:=u_{01, \lambda}=u_{02, \lambda} \quad \text { in } \quad \Omega,
$$

the unique solution of system $\left(P_{\lambda}\right)$ becomes $(u, v)=(w, w)$ where

$$
\begin{cases}\partial_{t} w_{\lambda}+(-\Delta+\lambda a(x, t)) w_{\lambda}=\alpha(x, t) w_{\lambda}+f_{\lambda} & \text { in } Q_{T}, \\ w_{\lambda}=0 & \text { in } \partial \Omega \times(0, T), \\ w_{\lambda}(x, 0)=w_{0, \lambda}(x) & \text { in } \Omega,\end{cases}
$$

reducing our model to one single equation setting. Thus, the analysis carried out here is equivalent to the results obtained in $[13,14,18]$ assuming one single equation. In [14], the potentials vanish in cylindrical domains and our results can be applied to similar problems as the ones considered there. On the other hand, [13] assumes more general non-cylindrical domains and the particular technical condition $\partial_{t} a_{i}(x, t) \leq 0$, a.e. in $(x, t) \in Q_{T}$, that we impose here to get the asymptotic behaviour of the problem (1.5), when the parameter $\lambda$ goes to infinity, falls into their setting for one single equation.

Our final result, see Sect. 6 , is a quantitative measure of the convergence of the components $u_{i, \lambda}$ in the regions where potentials $a_{i}$ are different from 0 , that is in $O_{a_{i}}^{c}$. More precisely, applying a Barry Simon's argument [20] we prove, under certain extra conditions on $a_{i}$ and $\alpha_{i}$ and assuming that $O_{a_{i}} \neq \emptyset$, that each component $u_{i, \lambda}$ decays exponentially fast to 0 with respect to $\lambda$ in the region $Q_{T} \backslash O_{a_{i}}$.

In fact, $\mathbf{U}_{\lambda}$ decays much faster than the standard estimate (1.3). Barry Simon's analysis was performed to give some partial answers for the semiclassical limit of the Schrödinger operator $\left(-h^{2} \Delta+V\right)$ in $\mathbb{R}^{N}$, when $V \geq 0$ is a $C^{\infty}$ potential bounded away from zero at infinity and has a finite number of non-degenerate zeros, multiple wells. Furthermore, he proved that the ground states concentrate into the set of zeros of the potential $V$, and either there is a rapid eigenvalue degeneracy or the limiting ground states reside asymptotically in a single well decaying exponentially to zero away from that well.

We have used these ideas to compute the exponentially decaying to zero in the regions where the potentials are away from zero. From an application point of view, the boundaries of the subdomains $O_{a_{i}}$ can be seen as potential barriers such as in Quantum mechanics. Moreover, at the limit $\lambda \rightarrow \infty$, the solution $\mathbf{U}$ will be zero everywhere apart from within the subdomains $O_{a_{i}}$, 
i.e., the solution cannot surpass those potential barriers. Furthermore, the decay outside the subdomains $O_{a_{i}}$ goes exponentially fast to zero.

We have also obtained a similar estimation for the stationary problem. We observe that the previous lemma can be particularised to a linear eigenvalue problem, i.e., $f_{i, \lambda}=\sigma u_{i, \lambda}$, so that we can get a decay estimate for the eigenfunctions on the boundary of vanishing regions $O_{a_{i}}$.

Finally, we might use our results in a future work to perform some numerical analysis of the non-cylindrical limiting problem $\left(P_{\infty}\right)$ that can be approximated by means of the cylindrical problem $\left(P_{\lambda}\right)$ for a big $\lambda$, which is simpler to handle. The strong convergence of the solutions along with the exponential decay shown provide us with with enough information to perform such an analysis.

\subsection{Notation}

The linear cooperative system $\left(P_{\lambda}\right)$ we are dealing with consists of two diffusive equations coupled through linear terms in the domain, each of them defined in $Q_{T}=\Omega \times(0, T)$. In this context, we will use, whenever it is possible, matrices and vectors to simplify the notation and we will keep the subindex $i$ to point out $i=1,2$, but we omit this clarification in what follows, when we focus on the components of the system.

We will use capital letters to denote vectors, while small letters to indicate the two components of the vector. Thus, let $\mathbf{U}=\left(u_{1}, u_{2}\right)^{T}$ be a vector composed by the two scalar functions $u_{i}$. We write $\mathbf{U}>0$ if $u_{1}>0$ and $u_{2}>0$ in the domain of definition.

We frequently use calligraphic letters to denote matrices. In particular, $\mathcal{L}$ will stand for "the laplacian matrix"

$$
\mathcal{L}=\left(\begin{array}{cc}
-\Delta & 0 \\
0 & -\Delta
\end{array}\right)
$$

and $\mathcal{P}_{\lambda}$ might be written as

$$
\mathcal{P}_{\lambda}:=\lambda \mathcal{A}-\mathcal{S}=\lambda\left(\begin{array}{cc}
a_{1}(x, t) & 0 \\
0 & a_{2}(x, t)
\end{array}\right)-\left(\begin{array}{cc}
0 & \alpha_{1}(x, t) \\
\alpha_{2}(x, t) & 0
\end{array}\right) ;
$$

see (1.1). Analogously to the vectors, $\mathcal{M}>0$ (or $\geq$ ) denotes a matrix whose entries are strictly positive (or non-negative) and we will say that $\mathcal{M}>\mathcal{N}$ if $\mathcal{M}-\mathcal{N}>0$ (respectively $<, \leq$ or $\geq$ ).

As for the functional spaces, in the sake of completeness and readability, we summarize here the main definitions that we are going to use. Let $\mathbf{L}^{2}(\Omega)$ be the space $L^{2}(\Omega) \times L^{2}(\Omega)$, equipped with the usual scalar product $\langle\boldsymbol{\Phi}, \boldsymbol{\Psi}\rangle_{\mathbf{L}^{2}(\Omega)}=\sum_{i}\left\langle\phi_{i}, \psi_{i}\right\rangle_{L^{2}(\Omega)}$ and the norm $\|\boldsymbol{\Phi}\|_{\mathbf{L}^{2}(\Omega)}^{2}=\sum_{i}\left\|\phi_{i}\right\|_{L^{2}(\Omega)}^{2}$. If we write $\boldsymbol{\Phi} \cdot \boldsymbol{\Psi}$ to denote the Euclidean scalar product of $\boldsymbol{\Phi}$ and $\boldsymbol{\Psi}$, we can express $\|\boldsymbol{\Phi}\|_{L^{2}(\Omega)}^{2}$ and $\langle\boldsymbol{\Phi}, \boldsymbol{\Psi}\rangle_{\mathbf{L}^{2}(\Omega)}$ as follows:

$$
\|\boldsymbol{\Phi}\|_{\mathbf{L}^{2}(\Omega)}=\langle\boldsymbol{\Phi}, \boldsymbol{\Phi}\rangle_{\mathbf{L}^{2}(\Omega)}=\int_{\Omega} \boldsymbol{\Phi} \cdot \boldsymbol{\Phi} \mathrm{d} x, \quad\langle\boldsymbol{\Phi}, \boldsymbol{\Psi}\rangle_{\mathbf{L}^{2}(\Omega)}=\int_{\Omega} \boldsymbol{\Phi} \cdot \boldsymbol{\Psi} \mathrm{d} x .
$$

To simplify the notation, we frequently use $\boldsymbol{\Phi}^{2}=\boldsymbol{\Phi} \cdot \boldsymbol{\Phi}$. Additionally, let $D \Phi$ be the $2 \times N$ Jacobian matrix. We denote the Frobenius inner product 
$D \boldsymbol{\Phi} \cdot D \boldsymbol{\Psi}$ between the matrices $D \boldsymbol{\Phi}$ and $D \boldsymbol{\Psi}$ as

$$
\begin{aligned}
D \boldsymbol{\Phi} \cdot D \boldsymbol{\Psi} & =\langle D \boldsymbol{\Phi}, D \boldsymbol{\Psi}\rangle_{F}=\operatorname{tr}\left(D \boldsymbol{\Phi}^{T} D \boldsymbol{\Psi}\right) \\
& =\sum_{i=1}^{2} \sum_{j=1}^{n} \frac{\partial \phi_{i}}{\partial x_{j}} \frac{\partial \psi_{i}}{\partial x_{j}}=\nabla \phi_{1} \cdot \nabla \psi_{1}+\nabla \phi_{2} \cdot \nabla \psi_{2},
\end{aligned}
$$

so that $|D \boldsymbol{\Phi}|^{2}=D \boldsymbol{\Phi} \cdot D \boldsymbol{\Phi}$. Consequently, the norm $\|D \boldsymbol{\Phi}\|_{\mathbf{L}^{2}(\Omega)}$ and the scalar product $\langle D \boldsymbol{\Phi}, D \boldsymbol{\Psi}\rangle_{\mathbf{L}^{2}(\Omega)}$ are

$$
\|D \boldsymbol{\Phi}\|_{\mathbf{L}^{2}(\Omega)}=\int_{\Omega}|D \boldsymbol{\Phi}|^{2} \mathrm{~d} x, \quad\langle D \boldsymbol{\Phi}, D \boldsymbol{\Psi}\rangle_{\mathbf{L}^{2}(\Omega)}=\int_{\Omega} D \boldsymbol{\Phi} \cdot D \boldsymbol{\Psi} \mathrm{d} x .
$$

Furthermore, we define the norm and the scalar product in $\mathbf{H}^{1}(\Omega)=H^{1}(\Omega) \times$ $H^{1}(\Omega)$ (see [17, Chapter 7]) through the expressions

$$
\begin{aligned}
\|\boldsymbol{\Phi}\|_{\mathbf{H}^{1}(\Omega)}^{2} & =\sum_{i}\left\|\phi_{i}\right\|_{L^{2}(\Omega)}^{2}+\left\|\nabla \phi_{i}\right\|_{L^{2}(\Omega)}^{2} \quad \text { and } \quad\langle\boldsymbol{\Phi}, \boldsymbol{\Psi}\rangle_{\mathbf{H}^{1}(\Omega)} \\
& =\sum_{i}\left\langle\phi_{i}, \psi_{i}\right\rangle_{L^{2}(\Omega)}+\left\langle\nabla \phi_{i}, \nabla \psi_{i}\right\rangle_{L^{2}(\Omega)},
\end{aligned}
$$

respectively. Therefore

$$
\begin{aligned}
\|\boldsymbol{\Phi}\|_{\mathbf{H}^{1}(\Omega)}^{2} & =\langle\boldsymbol{\Phi}, \boldsymbol{\Phi}\rangle_{\mathbf{H}^{1}(\Omega)}=\int_{\Omega} \boldsymbol{\Phi}^{2}+|D \boldsymbol{\Phi}|^{2} \mathrm{~d} x, \quad\langle\boldsymbol{\Phi}, \boldsymbol{\Psi}\rangle_{\mathbf{H}^{1}(\Omega)} \\
& =\int_{\Omega} \boldsymbol{\Phi} \cdot \boldsymbol{\Psi}+D \boldsymbol{\Phi} \cdot D \boldsymbol{\Psi} \mathrm{d} x .
\end{aligned}
$$

\section{The Stationary Problem}

Let $\Omega \subset \mathbb{R}^{N}$ be a bounded open domain and $a_{i}: \bar{\Omega} \rightarrow \mathbb{R}^{+} \cup\{0\}$, bounded non-negative measurable functions. We will focus on the stationary version of $\left(P_{\lambda}\right)$

$$
\begin{cases}\mathcal{L} \mathbf{U}_{\lambda}+\mathcal{P}_{\lambda} \mathbf{U}_{\lambda}=\mathbf{F}_{\lambda} & \text { in } \Omega \\ \mathbf{U}_{\lambda}=0 & \text { in } \partial \Omega .\end{cases}
$$

If $\mathbf{F}_{\lambda} \in \mathbf{L}^{2}(\Omega)$ is a family of pairs of functions depending on the real parameter $\lambda>0$ and uniformly bounded in $\mathbf{L}^{2}(\Omega)$, then existence and uniqueness of solutions $\mathbf{U}_{\lambda} \in \mathbf{H}_{0}^{1}(\Omega)$ to this problem yield form Lax-Milgram theorem, [15].

Moreover, we denote $K_{a_{i}}:=\left\{x \in \bar{\Omega} ; a_{i}(x)=0\right\} \subset \Omega$, are two closed sets in $\mathbb{R}^{N}$ and consider

$$
\Omega_{a_{i}}:=\operatorname{Int}\left(K_{a_{i}}\right) \neq \emptyset .
$$

Since $a_{i}$ are bounded in $\bar{\Omega}$ and the sets $K_{a_{i}}$ are closed in $\mathbb{R}^{n}$, we define the space $H_{0}^{1}\left(K_{a_{i}}\right)$ as follows, see [3]:

$$
H_{0}^{1}\left(K_{a_{i}}\right)=H^{1}\left(\mathbb{R}^{N}\right) \cap\left\{u_{i}=0 \text { a.e. in } \mathbb{R}^{N} \backslash K_{a_{i}}\right\},
$$

and, hence, thanks to the hypothesis (2.1), we find that $H_{0}^{1}\left(K_{a_{i}}\right) \neq\{0\}$. Observe that since we are considering closed sets we are working with a functional space of the form $H_{0}^{1}(A)$, where $A$ is a closed subdomain of $\mathbb{R}^{N}$. 
Hence, we cannot say that $H_{0}^{1}(A)=H_{0}^{1}(\operatorname{Int}(A))$, since to do so, we would need stronger assumptions on the regularity of the set $A$.

Let us also consider the limit problem, which will be later discussed in detail

$$
\begin{cases}\mathcal{L} \mathbf{U}=\mathbf{F} & \text { in } \Omega, \\ \mathbf{U}=0 & \text { in } \partial \Omega,\end{cases}
$$

where $\mathbf{U} \in H_{0}^{1}\left(K_{a_{1}}\right) \times H_{0}^{1}\left(K_{a_{2}}\right)$ and $\mathbf{F} \in \mathbf{L}^{2}(\Omega)$, where $\mathbf{F} \in \mathbf{L}^{2}(\Omega)$ is the limit of $\mathbf{F}_{\lambda}$ in the weak topology of $\mathbf{L}^{2}(\Omega)$, when $\lambda$ tends to $+\infty$.

Finally, given $\mathbf{V}$, we define the energy functional $E_{\lambda}$ in $\mathbf{L}^{2}(\Omega)$ as

$$
E_{\lambda}(\mathbf{V})= \begin{cases}\int_{\Omega}|D \mathbf{V}|^{2} \mathrm{~d} x+\lambda \int_{\Omega}(\mathcal{A} \mathbf{V}) \cdot \mathbf{V} \mathrm{d} x & \text { if } \mathbf{V} \in \mathbf{H}_{0}^{1}(\Omega), \\ +\infty & \text { otherwise }\end{cases}
$$

and the limit functional $E$ as

$$
E(\mathbf{V})= \begin{cases}\int_{\Omega}|D \mathbf{V}|^{2} \mathrm{~d} x & \text { if } \mathbf{V} \in H_{0}^{1}\left(K_{a_{1}}\right) \times H_{0}^{1}\left(K_{a_{2}}\right) \\ +\infty & \text { otherwise }\end{cases}
$$

Proposition 2.1. The solution $\mathbf{U}_{\lambda}$ of $\left(P_{\lambda}^{s}\right)$ is the unique minimiser in $\mathbf{H}_{0}^{1}(\Omega)$ for

$$
\mathbf{W} \mapsto I_{\lambda}(\mathbf{W}):=E_{\lambda}(\mathbf{W})-2 \int_{\Omega} \mathbf{F}_{\lambda} \cdot \mathbf{W} \mathrm{d} x-2 \int_{\Omega}\left(\mathcal{S} \mathbf{U}_{\lambda}\right) \cdot \mathbf{W} \mathrm{d} x
$$

Proof. Let $\mathbf{U}_{\lambda}$ be the unique solution of $\left(P_{\lambda}^{s}\right)$ and let $\mathbf{V}$ be any function belonging to $\mathbf{H}_{0}^{1}(\Omega)$. Then, $\mathbf{U}_{\lambda}-\mathbf{V}$ is an admissible test function in the weak formulation of $\left(P_{\lambda}^{s}\right)$, so that

$$
\begin{aligned}
0= & -\int_{\Omega}\left|D \mathbf{U}_{\lambda}\right|^{2} \mathrm{~d} x+\int_{\Omega} D \mathbf{U}_{\lambda} \cdot D \mathbf{V} \mathrm{d} x-\lambda \int_{\Omega}\left(\mathcal{A} \mathbf{U}_{\lambda}\right) \cdot \mathbf{U}_{\lambda} \mathrm{d} x \\
& +\lambda \int_{\Omega}\left(\mathcal{A} \mathbf{U}_{\lambda}\right) \cdot \mathbf{V} \mathrm{d} x+\int_{\Omega} \mathbf{F}_{\lambda} \cdot\left(\mathbf{U}_{\lambda}-\mathbf{V}\right) \mathrm{d} x+\int_{\Omega}\left(\mathcal{S} \mathbf{U}_{\lambda}\right) \cdot\left(\mathbf{U}_{\lambda}-\mathbf{V}\right) \mathrm{d} x .
\end{aligned}
$$

Subsequently, applying the Young's inequality, we arrive at one hand to

$$
\int_{\Omega} D \mathbf{U}_{\lambda} \cdot D \mathbf{V} \mathrm{d} x \leq \frac{1}{2} \int_{\Omega}|D \mathbf{U}|^{2} \mathrm{~d} x+\frac{1}{2} \int_{\Omega}|D \mathbf{V}|^{2} \mathrm{~d} x
$$

and on the other hand to

$$
\begin{aligned}
\lambda \int_{\Omega}\left(\mathcal{A} \mathbf{U}_{\lambda}\right) \cdot \mathbf{V} \mathrm{d} x= & \lambda \int_{\Omega}\left(\mathcal{A}^{\frac{1}{2}} \mathbf{U}_{\lambda}\right) \cdot\left(\mathcal{A}^{\frac{1}{2}} \mathbf{V}\right) \mathrm{d} x \leq \frac{\lambda}{2} \int_{\Omega}\left(\mathcal{A} \mathbf{U}_{\lambda}\right) \cdot \mathbf{U}_{\lambda} \mathrm{d} x \\
& +\frac{\lambda}{2} \int_{\Omega}(\mathcal{A} \mathbf{V}) \cdot \mathbf{V} \mathrm{d} x
\end{aligned}
$$


where $\mathcal{A}^{\frac{1}{2}}$ denotes the diagonal matrix with $a_{i}^{1 / 2}$ on the diagonal. Next, using (2.6) and (2.7) in (2.5), it yields

$$
\begin{aligned}
& \frac{1}{2} \int_{\Omega}\left|D \mathbf{U}_{\lambda}\right|^{2} \mathrm{~d} x+\frac{\lambda}{2} \int_{\Omega}\left(\mathcal{A} \mathbf{U}_{\lambda}\right) \cdot \mathbf{U}_{\lambda} \mathrm{d} x-\int_{\Omega} \mathbf{F}_{\lambda} \cdot \mathbf{U}_{\lambda} \mathrm{d} x-\int_{\Omega}\left(\mathcal{S} \mathbf{U}_{\lambda}\right) \cdot \mathbf{U}_{\lambda} \mathrm{d} x \\
& \quad \leq \frac{1}{2} \int_{\Omega}|D \mathbf{V}|^{2} \mathrm{~d} x+\frac{\lambda}{2} \int_{\Omega}(\mathcal{A} \mathbf{V}) \cdot \mathbf{V} \mathrm{d} x-\int_{\Omega} \mathbf{F}_{\lambda} \cdot \mathbf{V} \mathrm{d} x-\int_{\Omega}\left(\mathcal{S} \mathbf{U}_{\lambda}\right) \cdot \mathbf{V} \mathrm{d} x
\end{aligned}
$$

Thus, we can conclude that $\mathbf{U}_{\lambda}$ is a minimiser of (2.4).

To prove the uniqueness of the minimiser, we show that if $\mathbf{U}_{\lambda}$ minimises (2.4), then it is a solution of $\left(P_{\lambda}^{s}\right)$. To do so, let $\mathbf{U}_{\lambda}$ be a minimiser of $(2.4)$ and take any $\boldsymbol{\Phi} \in \mathbf{H}_{0}^{1}(\Omega)$. We define $j_{\lambda}(b):=I_{\lambda}\left(\mathbf{U}_{\lambda}+b \boldsymbol{\Phi}\right)$, for $b \in \mathbb{R}$ and $\mathbf{U}_{\lambda}+b \boldsymbol{\Phi} \in \mathbf{H}_{0}^{1}(\Omega)$, so that

$j_{\lambda}(b)=E_{\lambda}\left(\mathbf{U}_{\lambda}+b \boldsymbol{\Phi}\right)-2 \int_{\Omega} \mathbf{F}_{\lambda} \cdot\left(\mathbf{U}_{\lambda}+b \boldsymbol{\Phi}\right) \mathrm{d} x-2 \int_{\Omega}\left(\mathcal{S} \mathbf{U}_{\lambda}\right) \cdot\left(\mathbf{U}_{\lambda}+b \boldsymbol{\Phi}\right) \mathrm{d} x$.

The scalar function $j_{\lambda}(b)$ has a minimum at $b=0$ which implies that

$$
\begin{aligned}
0= & \left.\frac{\mathrm{d}\left(j_{\lambda}(b)\right)}{d b}\right|_{b=0}=\int_{\Omega} D \mathbf{U}_{\lambda} \cdot D \boldsymbol{\Phi} \mathrm{d} x+\lambda \int_{\Omega}\left(\mathcal{A} \mathbf{U}_{\lambda}\right) \cdot \boldsymbol{\Phi} \mathrm{d} x \\
& -\int_{\Omega} \mathbf{F}_{\lambda} \cdot \boldsymbol{\Phi} \mathrm{d} x-\int_{\Omega}\left(\mathcal{S} \mathbf{U}_{\lambda}\right) \cdot \boldsymbol{\Phi} \mathrm{d} x .
\end{aligned}
$$

The equality (2.9) is satisfied for any test function $\boldsymbol{\Phi} \in \mathbf{H}_{0}^{1}(\Omega)$. Therefore, it follows that $\mathbf{U}_{\lambda}$ is solution of $\left(P_{\lambda}^{s}\right)$ and, actually, the unique minimiser of $(2.4)$.

The analogous result holds for the limit problem.

Proposition 2.2. The solution $\mathbf{U}$ of $\left(P_{\infty}^{s}\right)$ is the unique minimiser in $H_{0}^{1}\left(K_{a_{1}}\right)$ $\times H_{0}^{1}\left(K_{a_{2}}\right)$ for

$$
\mathbf{W} \mapsto I(\mathbf{W}):=E(\mathbf{W})-2 \int_{\Omega} \mathbf{F} \cdot \mathbf{W} \mathrm{d} x .
$$

Proof. Let $\mathbf{U}$ be the unique solution of $\left(P_{\infty}^{s}\right)$ and let $\mathbf{Y} \in H_{0}^{1}\left(K_{a_{1}}\right) \times H_{0}^{1}\left(K_{a_{2}}\right)$. Then, $\mathbf{U}-\mathbf{Y}$ is an admissible test function in the weak formulation of $\left(P_{\infty}^{s}\right)$, so that

$$
0=-\int_{\Omega}|D \mathbf{U}|^{2} \mathrm{~d} x+\int_{\Omega} D \mathbf{U} \cdot D \mathbf{Y} \mathrm{d} x+\int_{\Omega} \mathbf{F} \cdot(\mathbf{U}-\mathbf{Y}) \mathrm{d} x .
$$

Note that the integration domain for each component $i$ is $K_{a_{i}}$ due to the geometry of the problem. Now, we apply Young's inequality, so that

$$
\frac{1}{2} \int_{\Omega}|D \mathbf{U}|^{2} \mathrm{~d} x-\int_{\Omega} \mathbf{F} \cdot \mathbf{U} \mathrm{d} x \leq \frac{1}{2} \int_{\Omega}|D \mathbf{Y}|^{2} \mathrm{~d} x-\int_{\Omega} \mathbf{F} \cdot \mathbf{Y} \mathrm{d} x .
$$

Therefore, (2.11) shows that $\mathbf{U}$ minimises (2.10).

The proof of uniqueness follows the same scheme as the proof of Proposition 2.1. We take here $\mathbf{U}$ as a minimiser of (2.10), $\boldsymbol{\Psi}$ any function in $H_{0}^{1}\left(K_{a_{1}}\right) \times H_{0}^{1}\left(K_{a_{2}}\right)$ and define for $b \in \mathbb{R}$ and $\mathbf{U}+b \boldsymbol{\Psi} \in H_{0}^{1}\left(K_{a_{1}}\right) \times H_{0}^{1}\left(K_{a_{2}}\right)$, the function $j(b)=I(\mathbf{U}+b \boldsymbol{\Psi})$. 
Proposition 2.3. The solution $\mathbf{U}_{\lambda}$ of $\left(P_{\lambda}^{s}\right)$ converges strongly in $\mathbf{H}^{1}(\Omega)$ when $\lambda \rightarrow+\infty$, to the unique solution $\mathbf{U}$ of $\left(P_{\infty}^{s}\right)$.

Proof. The proof is based on a standard application of the $\Gamma$-convergence for the functionals $E_{\lambda}$ and $E$; see [3] for further details.

For any $\lambda>0$, the solution $\mathbf{U}_{\lambda}$ of $\left(P_{\lambda}^{s}\right)$ is bounded in $\mathbf{L}^{2}(\Omega)$, since LaxMilgram theorem claims that $\mathbf{U}_{\lambda} \in \mathbf{H}_{0}^{1}(\Omega)$. We first prove that the sequence $\left\{\mathbf{U}_{\lambda}\right\}_{\lambda>0}$ is compact in $\mathbf{L}^{2}(\Omega)$. Indeed, $\mathbf{U}_{\lambda} \in \mathbf{H}_{0}^{1}(\Omega)$ is an admissible test function for the weak formulation of $\left(P_{\lambda}^{s}\right)$. Consequently

$$
\int_{\Omega}\left|D \mathbf{U}_{\lambda}\right|^{2} \mathrm{~d} x+\lambda \int_{\Omega}\left(\mathcal{A} \mathbf{U}_{\lambda}\right) \cdot \mathbf{U}_{\lambda} \mathrm{d} x=\int_{\Omega} \mathbf{F}_{\lambda} \cdot \mathbf{U}_{\lambda} \mathrm{d} x+\int_{\Omega}\left(\mathcal{S} \mathbf{U}_{\lambda}\right) \cdot \mathbf{U}_{\lambda} \mathrm{d} x .
$$

Using that $\mathbf{U}_{\lambda}$ is bounded in $\mathbf{L}^{2}(\Omega)$ together with the fact that the potentials $a_{i}$ are bounded measurable functions and applying Hölder's inequality, we get

$$
\int_{\Omega}\left|D \mathbf{U}_{\lambda}\right|^{2} \mathrm{~d} x \leq\left(\left\|\mathbf{F}_{\lambda}\right\|_{\mathbf{L}^{2}(\Omega)}+\left\|\alpha_{1}+\alpha_{2}\right\|_{L^{\infty}(\Omega)}\right)\left\|\mathbf{U}_{\lambda}\right\|_{\mathbf{L}^{2}(\Omega)} .
$$

Besides, since $\mathbf{U}_{\lambda} \in \mathbf{H}_{0}^{1}(\Omega)$, Poincaré's inequality claims that see [8, Corollary 9.19]

$$
\left\|\mathbf{U}_{\lambda}\right\|_{\mathbf{L}^{2}(\Omega)}^{2} \leq C\left\|D \mathbf{U}_{\lambda}\right\|_{\mathbf{L}^{2}(\Omega)}^{2}
$$

where $C$ depends on $\Omega$. As a result, the function $\mathbf{U}_{\lambda}$ is uniformly bounded in $\mathbf{H}_{0}^{1}(\Omega)$.

Let $\mathbf{V}$ be any point in the $\mathbf{L}^{2}(\Omega)$-adherence of the family $\left\{\mathbf{U}_{\lambda}\right\}_{\lambda>0}$. In other words, there exists a subsequence, still denoted by $\mathbf{U}_{\lambda}$, which converges in the strong topology of $\mathbf{L}^{2}(\Omega)$ to $\mathbf{V}$. Since $\mathbf{U}_{\lambda}$ is bounded in $\mathbf{H}_{0}^{1}(\Omega)$, we can assume without loss of generality that $\mathbf{U}_{\lambda}$ weakly converges in $\mathbf{H}_{0}^{1}(\Omega)$ to a function that necessarily must be $\mathbf{V}$.

Next, let $\mathbf{U} \in H_{0}^{1}\left(K_{a_{1}}\right) \times H_{0}^{1}\left(K_{a_{2}}\right)$ be the solution of the limit problem $\left(P_{\infty}^{s}\right)$. The sets $K_{a_{i}}$ are disjoint, which means that $u_{i}=0$ a.e. in $\Omega \backslash K_{a_{i}}$. In particular, by definition of this solution, we have that $\lambda a_{i} u_{i}=0$ a.e. in $\Omega$ and $E_{\lambda}(U)=E(U)$ for every $\lambda>0$. Recall that $\mathbf{U}_{\lambda}$ is the unique minimiser in $\mathbf{H}_{0}^{1}(\Omega)$ of the functional given by Eq. (2.4). Then

$$
\begin{aligned}
& E_{\lambda}\left(\mathbf{U}_{\lambda}\right)-2 \int_{\Omega} \mathbf{F}_{\lambda} \cdot \mathbf{U}_{\lambda} \mathrm{d} x-2 \int_{\Omega}\left(\mathcal{S} \mathbf{U}_{\lambda}\right) \cdot \mathbf{U}_{\lambda} \mathrm{d} x \leq E_{\lambda}(\mathbf{U}) \\
& \quad-2 \int_{\Omega} \mathbf{F}_{\lambda} \cdot \mathbf{U} \mathrm{d} x-2 \int_{\Omega}\left(\mathcal{S} \mathbf{U}_{\lambda}\right) \cdot \mathbf{U} \mathrm{d} x .
\end{aligned}
$$

Hence, letting $\lambda$ go to infinity in the previous inequality and taking into account Proposition 2.1, the lower semicontinuity of the functionals $E_{\lambda}$ and $E$ with respect to the weak convergence, and the $\Gamma$-convergence of the sequence $E_{\lambda}$ to $E$, see [3], it follows that:

$$
\begin{aligned}
E(\mathbf{V})-2 \int_{\Omega} \mathbf{F} \cdot \mathbf{V} \mathrm{d} x & \leq \liminf _{\lambda}\left(E_{\lambda}\left(\mathbf{U}_{\lambda}\right)-2 \int_{\Omega} \mathbf{F}_{\lambda} \cdot \mathbf{U}_{\lambda} \mathrm{d} x-2 \int_{\Omega}\left(\mathcal{S} \mathbf{U}_{\lambda}\right) \cdot \mathbf{U}_{\lambda} \mathrm{d} x\right) \\
& \leq \limsup _{\lambda}\left(E_{\lambda}\left(\mathbf{U}_{\lambda}\right)-2 \int_{\Omega} \mathbf{F}_{\lambda} \cdot \mathbf{U}_{\lambda} \mathrm{d} x-2 \int_{\Omega}\left(\mathcal{S} \mathbf{U}_{\lambda}\right) \cdot \mathbf{U}_{\lambda} \mathrm{d} x\right) \\
& \leq E(\mathbf{U})-2 \int_{\Omega} \mathbf{F} \cdot \mathbf{U} \mathrm{d} x
\end{aligned}
$$


which shows that $\mathbf{V}$ is a minimiser and thus $\mathbf{V}=\mathbf{U}$. By uniqueness of the adherence point, we infer that the whole sequence $\mathbf{U}_{\lambda}$ strongly converges in $\mathbf{L}^{2}(\Omega)$ to $\mathbf{U}$ and weakly in $\mathbf{H}_{0}^{1}(\Omega)$.

It remains to prove the strong convergence in $\mathbf{H}_{0}^{1}(\Omega)$. To do so, it is enough to prove

$$
\left\|D \mathbf{U}_{\lambda}\right\|_{\mathbf{L}^{2}(\Omega)} \rightarrow\|D \mathbf{U}\|_{\mathbf{L}^{2}(\Omega)}
$$

The weak convergence in $\mathbf{H}_{0}^{1}(\Omega)$ implies that $\|D \mathbf{U}\|_{\mathbf{L}^{2}(\Omega)} \leq \liminf \lambda_{\lambda}$ $\left\|D \mathbf{U}_{\lambda}\right\|_{\mathbf{L}^{2}(\Omega)}$. Therefore, (2.13) confirms that $\|D \mathbf{U}\|_{\mathbf{L}^{2}(\Omega)} \geq \limsup \operatorname{su}_{\lambda}$ $\left\|D \mathbf{U}_{\lambda}\right\|_{\mathbf{L}^{2}(\Omega)}$. Finally, the convergence of the whole sequence comes from uniqueness of the adherence point in $\mathbf{L}^{2}(\Omega)$.

As a consequence of Proposition 2.3, we easily obtain the following result.

Proposition 2.4. Assume that $\mathbf{F}_{\lambda}$ converges to $\mathbf{F}$ weakly in $\mathbf{L}^{2}(\Omega)$ and let $\mathbf{U}_{\lambda}$ be the solution of the problem $\left(P_{\lambda}^{s}\right)$. Then, when $\lambda \rightarrow \infty$

$$
\lambda \int_{\Omega}\left(\mathcal{A} \mathbf{U}_{\lambda}\right) \cdot \mathbf{U}_{\lambda} \mathrm{d} x \rightarrow 0
$$

Moreover, the convergence of $\lambda \mathcal{A} \mathbf{U}_{\lambda}$ holds in the $*$-weak topology of $\mathbf{H}^{-1}(\Omega):=$ $H^{-1}(\Omega) \times H^{1}(\Omega)$.

Proof. Due to Proposition 2.3, we know that $\mathbf{U}_{\lambda}$ strongly converges in $\mathbf{H}_{0}^{1}(\Omega)$ to $\mathbf{U}$, solution of $\left(P_{\infty}^{s}\right)$. Furthermore, $\mathbf{F}_{\lambda}$ weakly converges in $\mathbf{L}^{2}(\Omega)$ to a function $\mathbf{F} \in \mathbf{L}^{2}(\Omega)$. In particular

$$
\int_{\Omega}\left|D \mathbf{U}_{\lambda}\right|^{2} \mathrm{~d} x \rightarrow \int_{\Omega}|D \mathbf{U}|^{2} \mathrm{~d} x=\int_{\Omega} \mathbf{F} \cdot \mathbf{U} \mathrm{d} x, \quad \int_{\Omega} \mathbf{F}_{\lambda} \cdot \mathbf{U}_{\lambda} \mathrm{d} x \rightarrow \int_{\Omega} \mathbf{F} \cdot \mathbf{U} \mathrm{d} x .
$$

Now, passing to the limit, as $\lambda$ tends to infinity, in the expression

$$
\int_{\Omega}\left|D \mathbf{U}_{\lambda}\right|^{2} \mathrm{~d} x+\lambda \int_{\Omega}\left(\mathcal{A} \mathbf{U}_{\lambda}\right) \cdot \mathbf{U}_{\lambda} \mathrm{d} x=\int_{\Omega} \mathbf{F}_{\lambda} \cdot \mathbf{U}_{\lambda} \mathrm{d} x+\int_{\Omega}\left(\mathcal{S} \mathbf{U}_{\lambda}\right) \cdot \mathbf{U}_{\lambda} \mathrm{d} x,
$$

implies that

$$
\int_{\Omega}(\mathcal{S U}) \cdot \mathbf{U} \mathrm{d} x=0
$$

since $\mathbf{U} \in H_{0}^{1}\left(K_{a_{1}}\right) \times H_{0}^{1}\left(K_{a_{2}}\right)$. Thus, we obtain (2.14).

Next, let $\mathbf{U}_{\lambda}$ be solution of $\left(P_{\lambda}^{s}\right)$ and $\boldsymbol{\Phi} \in \mathbf{H}_{0}^{1}(\Omega)$ a test function. Once we integrate by parts in $\Omega$ we arrive at

$$
\int_{\Omega} \mathbf{U}_{\lambda} \cdot(\mathcal{L} \boldsymbol{\Phi}) \mathrm{d} x+\lambda \int_{\Omega}\left(\mathcal{A} \mathbf{U}_{\lambda}\right) \cdot \boldsymbol{\Phi} \mathrm{d} x=\int_{\Omega} \mathbf{F}_{\lambda} \cdot \boldsymbol{\Phi} \mathrm{d} x+\int_{\Omega}\left(\mathcal{S} \mathbf{U}_{\lambda}\right) \cdot \mathbf{U}_{\lambda} \mathrm{d} x .
$$

For each $\boldsymbol{\Phi}$ that satisfies $\|\boldsymbol{\Phi}\|_{\mathbf{H}^{1}(\Omega)} \leq 1$, we find

$$
\begin{aligned}
\left|\lambda \int_{\Omega}\left(\mathcal{A} \mathbf{U}_{\lambda}\right) \cdot \mathbf{\Phi} \mathrm{d} x\right| \leq & \left\|\mathbf{F}_{\lambda}\right\|_{\mathbf{L}^{2}(\Omega)}+\left\|D \mathbf{U}_{\lambda}\right\|_{\mathbf{L}^{2}(\Omega)} \\
& +\left\|\alpha_{1}+\alpha_{2}\right\|_{L^{\infty}(\Omega)}\left\|\mathbf{U}_{\lambda}\right\|_{\mathbf{L}^{2}(\Omega)} \leq C .
\end{aligned}
$$


Taking the supremum in $\boldsymbol{\Phi}$, we get

$$
\left|\lambda \int_{\Omega}\left(\mathcal{A} \mathbf{U}_{\lambda}\right) \cdot \boldsymbol{\Phi} \mathrm{d} x\right| \leq C .
$$

Therefore, the sequence $\lambda \mathcal{A} \mathbf{U}_{\lambda}$ is weakly-* sequentially compact in $\mathbf{H}^{-1}(\Omega)$ and we obtain the convergence by uniqueness of the limit in the distributional sense.

Remark 2.1. The estimation (2.15) will be improved in Sect. 6 performing an analysis which shows an exponential decay to zero of the solutions in the regions where the potentials are strictly positive. These results are mainly based on an argument performed by Barry Simon [20] in obtaining the semiclassical limit for an elliptic eigenvalue problem with potentials vanishing in multiple wells.

\section{Existence and Regularity of the Solutions of Problem $\left(P_{\lambda}\right)$}

In this very short section, we state the theorem that ensures existence and uniqueness of weak solution $\mathbf{U}_{\lambda}$ of problem $\left(P_{\lambda}\right)$. The proof is based on the well-known Galerkin method, which is rather standard for scalar problems, see [15], and easy to adapt to vector problems. Therefore, we give here just some details of how the proof would be.

Theorem 3.1. There exists a unique (weak) solution, $\mathbf{U}_{\lambda} \in \mathbf{H}_{0}^{1}\left(Q_{T}\right)$ of problem $\left(P_{\lambda}\right)$.

To apply Galerkin's method to get the existence of solutions, one might first construct solutions $\mathbf{U}_{\lambda, m}(t)$, with $u_{i, \lambda, m} \in L^{2}\left(0, T, H_{0}^{1}(\Omega)\right)$ of certain finite-dimensional approximations to $\left(P_{\lambda}\right)$ and, then, we pass to the limit. To do so, consider vector $\mathbf{W}_{k}:=\left(w_{1, k}, w_{2, k}\right)^{T}$, with $k \in \mathbb{N}$, so that $w_{i, k}$ only depend on $x$ and where $\left\{\mathbf{W}_{k}\right\}_{k=1}^{\infty}$ stands for an orthogonal basis in $\mathbf{H}_{0}^{1}(\Omega)$ and an orthonormal basis in $\mathbf{L}^{2}(\Omega)$, such that $u_{i, \lambda, m}(t):=\sum_{k=1}^{m} d_{i, k, m}(t) w_{i, k}$. In particular, we take $\left\{\mathbf{W}_{k}\right\}_{k=1}^{\infty}$ the complete set of normalised eigenfunctions of $\mathcal{L}$, under the decomposition (1.6) in $\mathbf{H}_{0}^{1}(\Omega)$. Actually, by the definition of the operator $\mathcal{L}$, we have pairs of eigenfunctions for each component of the operator $-\Delta$ in $\Omega$ with homogeneous Dirichlet boundary conditions.

The following remarks will play an important role in the subsequent analysis.

Remark 3.1. (Theorem 3, Chapter 5.9, [15]). If $\mathbf{U} \in \mathbf{H}_{0}^{1}\left(Q_{T}\right)$ and $\partial_{t} \mathbf{U} \in$ $\mathbf{H}^{-1}\left(Q_{T}\right):=L^{2}\left(0, T, H_{0}^{-1}(\Omega)\right) \times L^{2}\left(0, T, H_{0}^{-1}(\Omega)\right)$, then

$$
\mathbf{U} \in C\left([0, T], L^{2}(\Omega)\right) \times C\left([0, T], L^{2}(\Omega)\right),
$$

after possibly being redefined on a set of measure zero. Moreover, the mapping $t \mapsto\|\mathbf{U}(t)\|_{\mathbf{L}^{2}(\Omega)}^{2}$ is absolutely continuous and

$$
\frac{\mathrm{d}}{\mathrm{d} t}\|\mathbf{U}(t)\|_{\mathbf{L}^{2}(\Omega)}^{2}=2\left\langle\partial_{t} \mathbf{U}(t), \mathbf{U}(t)\right\rangle_{\mathbf{L}^{2}(\Omega)} .
$$


Remark 3.2. (Improved regularity, [15]). Let $\mathbf{U}_{0, \lambda} \in \mathbf{H}^{2}(\Omega):=H^{2}(\Omega) \times$ $H^{2}(\Omega), \partial_{t} \mathbf{F}_{\lambda} \in \mathbf{L}^{2}\left(Q_{T}\right)$ and let $\mathbf{U}_{\lambda}$ be the weak solution of $\left(P_{\lambda}\right)$. Then

$$
\begin{aligned}
& \mathbf{U}_{\lambda} \in L^{\infty}\left(0, T ; H^{2}(\Omega)\right) \times L^{\infty}\left(0, T ; H^{2}(\Omega)\right), \\
& \partial_{t} \mathbf{U}_{\lambda} \in L^{\infty}\left(0, T ; L^{2}(\Omega)\right) \cap L^{2}\left(0, T, H_{0}^{1}(\Omega)\right) \times L^{\infty}\left(0, T ; L^{2}(\Omega)\right) \cap L^{2}\left(0, T, H_{0}^{1}(\Omega)\right), \\
& \partial_{t}^{2} \mathbf{U}_{\lambda} \in \mathbf{H}^{-1}\left(Q_{T}\right) .
\end{aligned}
$$

\section{Uniqueness of the Solution of the Limiting Problem}

We study in this section the uniqueness of solutions to the problem that emerges as the limit of $\left(P_{\lambda}\right)$ when $\lambda$ tends to infinity. In Sect. 5.2, we prove the existence of the solution as the limit of the solutions of $\left(P_{\lambda}\right)$.

To this aim, let $\boldsymbol{\Phi} \in \mathbf{H}_{0}^{1}\left(Q_{T}\right)$ be a test function and consider the weak formulation of $\left(P_{\lambda}\right)$

$$
\int_{Q_{T}}\left(\partial_{t} \mathbf{U} \cdot \mathbf{\Phi}+D \mathbf{U} \cdot D \boldsymbol{\Phi}+\lambda(\mathcal{A} \mathbf{U}) \cdot \mathbf{\Phi}-(\mathcal{S U}) \cdot \boldsymbol{\Phi}\right) \mathrm{d} x \mathrm{~d} t=\int_{Q_{T}} \mathbf{F} \cdot \boldsymbol{\Phi} \mathrm{d} x \mathrm{~d} t
$$

we get the limit problem for this equation, at least formally, by noting that, as $\lambda \rightarrow \infty$, thanks to (1.3), the solution concentrates in the regions where $a_{i}$ vanish, and is equal to zero everywhere else, so that the term $\lambda(\mathcal{A} \mathbf{U}) \cdot \boldsymbol{\Phi}$ disappears. Hence, since in the limit $u_{i}=\phi_{i}=0$ a.e. in $Q_{T} \backslash O_{a_{i}}$ and due to the geometrical structure (1.2), we get that $\int_{Q_{T}}(\mathcal{S U}) \cdot \boldsymbol{\Phi} \mathrm{d} x \mathrm{~d} t$ cancels out.

Therefore, we consider

$$
\left\{\begin{array}{l}
\int_{Q_{T}}\left(\partial_{t} \mathbf{U} \cdot \boldsymbol{\Phi}+D \mathbf{U} \cdot D \boldsymbol{\Phi}\right) \mathrm{d} x \mathrm{~d} t=\int_{Q_{T}} \mathbf{F} \cdot \boldsymbol{\Phi} \mathrm{d} x \mathrm{~d} t \\
u_{i}=\phi_{i}=0 \text { a.e. in } Q_{T} \backslash O_{a_{i}} \\
\mathbf{U}(x, 0)=\mathbf{U}_{0} \in \mathbf{H}_{0}^{1}(\Omega) .
\end{array}\right.
$$

which will turn out to be, as we will see in the next section, the limit problem of $\left(P_{\lambda}\right)$.

Observe that Remark 3.1 establishes that solution $\mathbf{U}$ of $\left(P_{\infty}\right)$ is continuous in time. Therefore, the initial condition $\mathbf{U}_{0}(x)$ makes sense in $\mathbf{L}^{2}(\Omega)$. Moreover, Galerkin's method provides us with the following regularity of the solutions for $\mathbf{F} \in \mathbf{L}^{2}\left(Q_{T}\right)$ and $\mathbf{U}_{0}=\in \mathbf{H}_{0}^{1}(\Omega)$ :

$$
\mathbf{U} \in \mathbf{H}^{1}\left(Q_{T}\right), \quad \partial_{t} \mathbf{U} \in \mathbf{H}^{-1}\left(Q_{T}\right) .
$$

Proposition 4.1. Let $\mathbf{U}$ be a solution of $\left(P_{\infty}\right)$. Then

$$
\frac{1}{4} \sup _{t \in(0, T)}\|\mathbf{U}(t)\|_{\mathbf{L}^{2}(\Omega)}^{2}+\|D \mathbf{U}\|_{\mathbf{L}^{2}\left(Q_{T}\right)}^{2} \leq \frac{1}{2}\left\|\mathbf{U}_{0}\right\|_{\mathbf{L}^{2}(\Omega)}^{2}+T\|\mathbf{F}\|_{\mathbf{L}^{2}\left(Q_{T}\right)}^{2} .
$$

Proof. Let $\mathbf{U}$ be a solution of $\left(P_{\infty}\right)$, and $s \in(0, T)$. Define $Q_{S}=\Omega \times(0, s)$. Choose $\boldsymbol{\Phi}=\mathbf{U} \mathbf{1}_{[0, s]}$, where $\mathbf{1}_{[0, s]}$ is the characteristic function of $(0, s)$, as test function in $\left(P_{\infty}\right)$

$$
\int_{Q_{S}} \partial_{t} \mathbf{U} \cdot \mathbf{U} \mathrm{d} x \mathrm{~d} t+\int_{Q_{S}}|D \mathbf{U}|^{2} \mathrm{~d} x \mathrm{~d} t=\int_{Q_{S}} \mathbf{F} \cdot \mathbf{U} \mathrm{d} x \mathrm{~d} t .
$$


Thanks to Remark 3.1 and taking into account the regularity of $\mathbf{U}$ and $\partial_{t} \mathbf{U}$, it follows that $t \mapsto\|\mathbf{U}(t)\|_{\mathbf{L}^{2}(\Omega)}^{2}$ is absolutely continuous and hence:

$$
\frac{\mathrm{d}}{\mathrm{d} t}\|\mathbf{U}(t)\|_{\mathbf{L}^{2}(\Omega)}^{2}=2\left\langle\partial_{t} \mathbf{U}(t), \mathbf{U}(\mathbf{t})\right\rangle_{\mathbf{L}^{2}(\Omega)},
$$

for a.e. $0 \leq t \leq T$. Therefore, we get

$$
\frac{1}{2}\|\mathbf{U}(s)\|_{\mathbf{L}^{2}(\Omega)}-\frac{1}{2}\|\mathbf{U}(0)\|_{\mathbf{L}^{2}(\Omega)}+\int_{Q_{S}}|D \mathbf{U}| \mathrm{d} x \mathrm{~d} t=\int_{Q_{S}} \mathbf{F} \cdot \mathbf{U} \mathrm{d} x \mathrm{~d} t .
$$

Moreover, Young's inequality and the Integral Mean Value Theorem imply that

$$
\begin{aligned}
\left|\int_{Q_{S}} \mathbf{F} \cdot \mathbf{U} \mathrm{d} x \mathrm{~d} t\right| & \leq \frac{k}{2}\|\mathbf{F}\|_{\mathbf{L}^{2}\left(Q_{S}\right)}^{2}+\frac{1}{2 k} \int_{Q_{S}} \mathbf{U}^{2} \mathrm{~d} x \mathrm{~d} t \\
& \leq \frac{k}{2}\|\mathbf{F}\|_{\mathbf{L}^{2}\left(Q_{S}\right)}^{2}+\frac{T}{2 k} \sup _{t \in(0, T)}\|\mathbf{U}\|_{\mathbf{L}^{2}(\Omega)}^{2} .
\end{aligned}
$$

Indeed, if we set $k=2 T$

$$
\begin{aligned}
\frac{1}{2}\|\mathbf{U}(s)\|_{\mathbf{L}^{2}(\Omega)}^{2}+\int_{Q_{S}}|D \mathbf{U}|^{2} \mathrm{~d} x \mathrm{~d} t \leq & \frac{1}{2}\left\|\mathbf{U}_{0}\right\|_{\mathbf{L}^{2}(\Omega)}^{2}+T\|\mathbf{F}\|_{\mathbf{L}^{2}\left(Q_{S}\right)}^{2} \\
& +\frac{1}{4} \sup _{t \in(0, T)}\|\mathbf{U}(t)\|_{\mathbf{L}^{2}(\Omega)}^{2} .
\end{aligned}
$$

The previous calculations are true for every $s \in(0, T)$. Thus, taking the supremum in $s \in(0, T)$, we get (4.1).

Corollary 4.2. There exists at most a unique solution $\mathbf{U}$ of $\left(P_{\infty}\right)$.

Proof. Assume that $\mathbf{U}_{1}$ and $\mathbf{U}_{2}$ are two different solutions of $\left(P_{\infty}\right)$ and define $\mathbf{Y}:=\mathbf{U}_{1}-\mathbf{U}_{2}$. Then, $\mathbf{Y}$ is also a solution of $\left(P_{\infty}\right)$ with $\mathbf{F}=0$ and $\mathbf{U}_{0}=0$. Consequently, if we insert $\mathbf{Y}$ into Eq. (4.1) turns out that $\mathbf{Y}=0$, which implies the uniqueness of the solution of $\left(P_{\infty}\right)$.

\section{Asymptotic Behaviour as $\lambda$ Goes to Infinity}

\subsection{Energy Bounds}

The convergence of $\mathbf{U}_{\lambda}$ to $\mathbf{U}$, solution of $\left(P_{\infty}\right)$, comes from a series of uniform energy estimates in $\lambda$ for $\mathbf{U}_{\lambda}$ and $\partial_{t} \mathbf{U}_{\lambda}$. In particular, if we use $\mathbf{U}_{\lambda}$ as a test function in the weak formulation of $\left(P_{\lambda}\right)$, we obtain a bound that guarantees that $u_{i}=0$ a.e. in $O_{a_{i}}$.

On the other hand, $\partial_{t} \mathbf{U}_{\lambda}$ belongs to $\mathbf{H}_{0}^{1}\left(Q_{T}\right)$ if $\mathbf{U}_{0, \lambda} \in \mathbf{H}^{2}(\Omega)$ and $\partial_{t} \mathbf{F}_{\lambda} \in \mathbf{L}^{2}\left(Q_{T}\right)$, according to Remark 3.2. Under these assumptions, we can use $\partial_{t} \mathbf{U}_{\lambda}$ as a test function in the weak formulation of $\left(P_{\lambda}\right)$ to find a bound which claims that $\left\|\partial_{t} \mathbf{U}_{\lambda}\right\|_{\mathbf{L}^{2}\left(Q_{T}\right)} \leq C$ if we assume that the potentials $a_{i}$ are time decreasing and satisfy the following condition:

$$
\sup _{\lambda}\left(\lambda \int_{\Omega}\left(\mathcal{A}(x, 0) \mathbf{U}_{0, \lambda}\right) \cdot \mathbf{U}_{0, \lambda} \mathrm{d} x\right)<\infty,
$$

where $\mathbf{U}_{0, \lambda}$ is a bounded sequence in $\mathbf{H}_{0}^{1}(\Omega)$. 
Lemma 5.1. Let $\mathbf{U}_{\lambda}$ be the weak solution of problem $\left(P_{\lambda}\right)$ with $\mathbf{U}_{0, \lambda} \in \mathbf{H}_{0}^{1}(\Omega)$ the initial condition and $\mathbf{F}_{\lambda} \in \mathbf{L}^{2}\left(Q_{T}\right)$. Then

$$
\begin{aligned}
& \frac{1}{4} \sup _{t \in(0, T)}\left\|\mathbf{U}_{\lambda}(t)\right\|_{\mathbf{L}^{2}(\Omega)}^{2}+\left\|D \mathbf{U}_{\lambda}\right\|_{\mathbf{L}^{2}\left(Q_{T}\right)}^{2}+\lambda \int_{Q_{T}}\left(\mathcal{A}_{\lambda} \mathbf{U}_{\lambda}\right) \cdot \mathbf{U}_{\lambda} \mathrm{d} x \mathrm{~d} t \\
& \quad \leq \frac{1}{2}\left\|\mathbf{U}_{0, \lambda}\right\|_{\mathbf{L}^{2}(\Omega)}^{2}+T\left\|\mathbf{F}_{\lambda}\right\|_{\mathbf{L}^{2}\left(Q_{T}\right)}^{2}+\sup _{Q_{T}}\left\{\left\|\alpha_{1}\right\|_{L^{\infty}\left(Q_{T}\right)},\left\|\alpha_{2}\right\|_{L^{\infty}\left(Q_{T}\right)}\right\}\left\|\mathbf{U}_{\lambda}\right\|_{\mathbf{L}^{2}\left(Q_{T}\right)},
\end{aligned}
$$

where $L^{\infty}\left(Q_{T}\right)=L^{\infty}\left(0, T, L^{\infty}(\Omega)\right)$.

Proof. Let $\mathbf{U}_{\lambda}$ be the solution of $\left(P_{\lambda}\right)$ and $s \in(0, T)$ and let $\boldsymbol{\Phi}=\mathbf{U}_{\lambda} \mathbf{1}_{[0, s]}$ be a test function. Then, the weak formulation of $\left(P_{\lambda}\right)$ yields

$$
\begin{aligned}
\frac{1}{2}\left\|\mathbf{U}_{\lambda}(s)\right\|_{\mathbf{L}^{2}(\Omega)}^{2}-\frac{1}{2}\left\|\mathbf{U}_{\lambda}(0)\right\|_{\mathbf{L}^{2}(\Omega)}^{2}+\int_{Q_{S}}\left|D \mathbf{U}_{\lambda}\right|^{2} \mathrm{~d} x \mathrm{~d} t+\lambda \int_{Q_{T}}\left(\mathcal{A} \mathbf{U}_{\lambda}\right) \cdot \mathbf{U}_{\lambda} \mathrm{d} x \mathrm{~d} t \\
=\int_{Q_{S}} \mathbf{F}_{\lambda} \cdot \mathbf{U}_{\lambda} \mathrm{d} x \mathrm{~d} t+\int_{Q_{T}}\left(\mathcal{S} \mathbf{U}_{\lambda}\right) \cdot \mathbf{U}_{\lambda} \mathrm{d} x \mathrm{~d} t .
\end{aligned}
$$

Repeating the same procedure as in the proof of Proposition 4.1, and knowing that all coefficients $\alpha_{1}$ and $\alpha_{2}$ are uniformly bounded, once we apply Hölder's inequality, we obtain equation (5.1), so we omit the details.

Next, we want to find an uniform bound for $\left\|\partial_{t} \mathbf{U}_{\lambda}\right\|_{\mathbf{L}^{2}\left(Q_{T}\right)}$. To this aim, we must assume a time monotonicity condition for the potentials $a_{i}$.

Definition 5.2. We say that the functions $a_{i}: Q_{T} \rightarrow \mathbb{R}^{+} \cup\{0\}$ satisfy a time decay condition in $Q_{T}$ if they are Lipschitz and

$$
\partial_{t} a_{i}(x, t) \leq 0, \text { a.e. in }(x, t) \in Q_{T} .
$$

Lemma 5.3. Let the potentials $a_{i}$ satisfy a time decay condition in $Q_{T}, \mathbf{U}_{0, \lambda} \in$ $\mathbf{H}^{2}(\Omega)$ and $\partial_{t} \mathbf{F}_{\lambda} \in \mathbf{L}^{2}\left(Q_{T}\right)$. Then, the solution $\mathbf{U}_{\lambda}$ of $\left(P_{\lambda}\right)$ satisfies

$$
\begin{aligned}
& (1-\epsilon) \int_{Q_{T}}\left(\partial_{t} \mathbf{U}_{\lambda}\right)^{2} \mathrm{~d} x \mathrm{~d} t+\sup _{s \in(0, T)}\left(\int_{\Omega}\left|D \mathbf{U}_{\lambda}(s)\right|^{2} \mathrm{~d} x\right) \\
& \leq \int_{Q_{T}} \mathbf{F}_{\lambda}^{2} \mathrm{~d} x \mathrm{~d} t+\int_{\Omega}\left|D \mathbf{U}_{0, \lambda}\right|^{2} \mathrm{~d} x+\frac{\xi}{2 \epsilon}\left\|\mathbf{U}_{\lambda}\right\|_{\mathbf{L}^{2}\left(Q_{T}\right)}^{2} \\
& \quad+\lambda \int_{\Omega}\left(\mathcal{A}(x, 0) \mathbf{U}_{\lambda}(0)\right) \cdot \mathbf{U}_{\lambda}(0) \mathrm{d} x,
\end{aligned}
$$

for $\epsilon>0$ sufficiently small and $\xi=\max _{Q_{T}}\left\{\left\|\alpha_{1}\right\|_{L^{\infty}\left(Q_{T}\right)},\left\|\alpha_{2}\right\|_{L^{\infty}\left(Q_{T}\right)}\right\}$.

Proof. Remark 3.2 implies that $\partial_{t} \mathbf{U}_{\lambda} \in \mathbf{H}_{0}^{1}(\Omega)$. Thus, for each $s \in(0, T)$, $\boldsymbol{\Phi}=\partial_{t} \mathbf{U}_{\lambda} \mathbf{1}_{[0, s]}$ is an admissible test function in the weak formulation of $\left(P_{\lambda}\right)$. In fact, we get

$$
\begin{aligned}
& \int_{Q_{S}}\left(\partial_{t} \mathbf{U}_{\lambda}\right)^{2} \mathrm{~d} x \mathrm{~d} t+\int_{Q_{S}} D \mathbf{U}_{\lambda} \cdot D\left(\partial_{t} \mathbf{U}_{\lambda}\right) \mathrm{d} x \mathrm{~d} t \\
& +\int_{Q_{S}}\left(\mathcal{P}_{\lambda} \mathbf{U}_{\lambda}\right) \cdot \partial_{t} \mathbf{U}_{\lambda} \mathrm{d} x \mathrm{~d} t=\int_{Q_{S}} \mathbf{F}_{\lambda} \cdot \partial_{t} \mathbf{U}_{\lambda} \mathrm{d} x \mathrm{~d} t .
\end{aligned}
$$


Moreover, since $\nabla u_{i, \lambda} \in L^{2}\left(0, T, H^{1}(\Omega)\right)$ and $\nabla\left(\partial_{t} u_{i, \lambda}\right) \in L^{2}\left(0, T, L^{2}(\Omega)\right)$, thanks to Remark 3.1 and Fubini's theorem

$$
\int_{Q_{S}} D \mathbf{U}_{\lambda} \cdot D\left(\partial_{t} \mathbf{U}_{\lambda}\right) \mathrm{d} x \mathrm{~d} t=\frac{1}{2} \int_{0}^{s} \frac{\mathrm{d}}{\mathrm{d} t}\left(\int_{\Omega}\left|D \mathbf{U}_{\lambda}\right|^{2} \mathrm{~d} x\right) \mathrm{d} t
$$

and

$$
\begin{aligned}
\lambda \int_{Q_{S}}\left(\mathcal{A} \mathbf{U}_{\lambda}\right) \cdot \partial_{t} \mathbf{U}_{\lambda} \mathrm{d} x \mathrm{~d} t= & \lambda \int_{0}^{s}\left(\frac{\mathrm{d}}{\mathrm{d} t}\left(\frac{1}{2} \int_{\Omega}\left(\mathcal{A} \mathbf{U}_{\lambda}\right) \cdot \mathbf{U}_{\lambda} \mathrm{d} x\right)\right. \\
& \left.-\frac{1}{2} \int_{\Omega}\left(\mathcal{A}^{\prime} \mathbf{U}_{\lambda}\right) \cdot \mathbf{U}_{\lambda} \mathrm{d} x\right) \mathrm{d} t
\end{aligned}
$$

such that

$$
\mathcal{A}^{\prime}=\left(\begin{array}{cc}
a_{1}^{\prime} & 0 \\
0 & a_{2}^{\prime}
\end{array}\right), \quad a_{i}^{\prime}=\frac{\partial a_{i}(x, t)}{\partial t} .
$$

If we substitute the above expressions into (5.4), we arrive at

$$
\begin{aligned}
\int_{Q_{S}} & \left(\partial_{t} \mathbf{U}_{\lambda}\right)^{2} \mathrm{~d} x \mathrm{~d} t+\frac{1}{2} \int_{\Omega}\left|D \mathbf{U}_{\lambda}(s)\right|^{2} \mathrm{~d} x+\frac{\lambda}{2} \int_{\Omega}\left(\mathcal{A}(x, s) \mathbf{U}_{\lambda}(s)\right) \cdot \mathbf{U}_{\lambda}(s) \mathrm{d} x \\
& -\frac{\lambda}{2} \int_{Q_{S}}\left(\mathcal{A}^{\prime} \mathbf{U}_{\lambda}\right) \cdot \mathbf{U}_{\lambda} \mathrm{d} x \mathrm{~d} t \\
= & \int_{Q_{S}} \mathbf{F}_{\lambda} \cdot \partial_{t} \mathbf{U}_{\lambda} \mathrm{d} x \mathrm{~d} t+\int_{Q_{S}}\left(\mathcal{S} \mathbf{U}_{\lambda}\right) \cdot \partial_{t} \mathbf{U}_{\lambda} \mathrm{d} x \mathrm{~d} t+\frac{1}{2} \int_{\Omega}\left|D \mathbf{U}_{\lambda}(0)\right|^{2} \mathrm{~d} x \\
& +\frac{\lambda}{2} \int_{\Omega}\left(\mathcal{A}(x, 0) \mathbf{U}_{\lambda}(0)\right) \cdot \mathbf{U}_{\lambda}(0) \mathrm{d} x .
\end{aligned}
$$

Applying Young's inequality, it yields

$$
\int_{Q_{S}} \mathbf{F}_{\lambda} \cdot \partial_{t} \mathbf{U}_{\lambda} \mathrm{d} x \mathrm{~d} t \leq \frac{1}{2} \int_{Q_{S}} \mathbf{F}_{\lambda}^{2} \mathrm{~d} x \mathrm{~d} t+\frac{1}{2} \int_{Q_{S}}\left(\partial_{t} \mathbf{U}_{\lambda}\right)^{2} \mathrm{~d} x \mathrm{~d} t,
$$

and

$$
\int_{Q_{S}}\left(\mathcal{S} \mathbf{U}_{\lambda}\right) \cdot \partial_{t} \mathbf{U}_{\lambda} \mathrm{d} x \mathrm{~d} t \leq \frac{1}{2 \epsilon} \int_{Q_{S}}\left(\mathcal{S} \mathbf{U}_{\lambda}\right)^{2} \mathrm{~d} x \mathrm{~d} t+\frac{\epsilon}{2} \int_{Q_{S}}\left(\partial_{t} \mathbf{U}_{\lambda}\right)^{2} \mathrm{~d} x \mathrm{~d} t
$$

for a sufficiently small $\epsilon>0$. Therefore, we have

$$
\begin{aligned}
(1- & \epsilon) \int_{Q_{S}}\left(\partial_{t} \mathbf{U}_{\lambda}\right)^{2} \mathrm{~d} x \mathrm{~d} t+\int_{\Omega}\left|D \mathbf{U}_{\lambda}(s)\right|^{2} \mathrm{~d} x+\lambda \int_{\Omega}\left(\mathcal{A}(x, s) \mathbf{U}_{\lambda}(s)\right) \cdot \mathbf{U}_{\lambda}(s) \mathrm{d} x \\
& -\lambda \int_{Q_{S}}\left(\mathcal{A}^{\prime} \mathbf{U}_{\lambda}\right) \cdot \mathbf{U}_{\lambda} \mathrm{d} x \mathrm{~d} t \\
\leq & \int_{Q_{S}} \mathbf{F}_{\lambda}^{2} \mathrm{~d} x \mathrm{~d} t+\frac{1}{\epsilon} \int_{Q_{S}}\left[\left(\alpha_{1} u_{2, \lambda}\right)^{2}+\left(\alpha_{2} u_{1, \lambda}\right)^{2}\right] \mathrm{d} x \mathrm{~d} t+\int_{\Omega}\left|D \mathbf{U}_{\lambda}(0)\right|^{2} \mathrm{~d} x \\
& +\lambda \int_{\Omega}\left(\mathcal{A}(x, 0) \mathbf{U}_{\lambda}(0)\right) \cdot \mathbf{U}_{\lambda}(0) \mathrm{d} x .
\end{aligned}
$$

Finally, using Definition 5.2, we conclude that

$$
(1-\epsilon) \int_{Q_{S}}\left(\partial_{t} \mathbf{U}_{\lambda}\right)^{2} \mathrm{~d} x \mathrm{~d} t+\int_{\Omega}\left|D \mathbf{U}_{\lambda}(s)\right|^{2} \mathrm{~d} x
$$




$$
\begin{aligned}
\leq & \int_{Q_{S}} \mathbf{F}_{\lambda}^{2} \mathrm{~d} x \mathrm{~d} t+\frac{1}{\epsilon} \int_{Q_{S}}\left(\mathcal{S} \mathbf{U}_{\lambda}\right)^{2} \mathrm{~d} x \mathrm{~d} t+\int_{\Omega}\left|D \mathbf{U}_{0, \lambda}\right|^{2} \mathrm{~d} x \\
& +\lambda \int_{\Omega}\left(\mathcal{A}(x, 0) \mathbf{U}_{\lambda}(0)\right) \cdot \mathbf{U}_{\lambda}(0) \mathrm{d} x .
\end{aligned}
$$

Taking the supremum in $s$ and applying Hölder's inequality we arrive at (5.3).

\subsection{Convergence of Weak Solutions}

Using the previous estimation results, we prove the weak convergence of $\mathbf{U}_{\lambda}$ to $\mathbf{U}$, solution of problem $\left(P_{\infty}\right)$, under the assumption that $a_{i}$ satisfy a time decay condition in $Q_{T}$ and imposing certain additional conditions.

Let $\mathbf{F}_{\lambda}$ be a bounded sequence in $\mathbf{L}^{2}\left(Q_{T}\right), \mathbf{U}_{0, \lambda}$ a bounded sequence in $\mathbf{H}_{0}^{1}(\Omega)$, and suppose that $\partial_{t} \mathbf{F}_{\lambda} \in \mathbf{L}^{2}\left(Q_{T}\right), \mathbf{U}_{0, \lambda} \in \mathbf{H}^{2}(\Omega)$. Moreover, assume that $\mathbf{F}_{\lambda}$ weakly converges to $\mathbf{F}$ in $\mathbf{L}^{2}\left(Q_{T}\right)$ and $\mathbf{U}_{0, \lambda}$ weakly converges to $\mathbf{U}_{0} \in \mathbf{H}_{0}^{1}(\Omega)$, at least for a subsequence.

Proposition 5.4. Suppose that potentials $a_{i}$ satisfy a time decay condition in $Q_{T}$ and that

$$
\sup _{\lambda}\left(\lambda \int_{\Omega}\left(\mathcal{A}(x, 0) \mathbf{U}_{0, \lambda}\right) \cdot \mathbf{U}_{0, \lambda} \mathrm{d} x\right)<\infty .
$$

Furthermore, let $\mathbf{U}_{\lambda}$ be a solution of problem $\left(P_{\lambda}\right)$. Then, when $\lambda$ tends to infinity, $\mathbf{U}_{\lambda}$ weakly converges in $\mathbf{H}_{0}^{1}\left(Q_{T}\right)$ to $\mathbf{U}$, the unique solution of problem $\left(P_{\infty}\right)$.

Proof. Due to Lemma 5.1, we know that $\mathbf{U}_{\lambda}$ is uniformly bounded in $\mathbf{H}_{0}^{1}\left(Q_{T}\right)$. Consequently, it weakly converges in $\mathbf{H}_{0}^{1}\left(Q_{T}\right)$ to a function $\mathbf{U} \in \mathbf{H}_{0}^{1}\left(Q_{T}\right)$. Simultaneously, $\nabla u_{i, \lambda}$ weakly converges to $\nabla u_{i}$ in $L^{2}\left(0, T, L^{2}(\Omega)\right)$. Assuming the time decay of $a_{i}(5.2)$, and since $\mathbf{U}_{0, \lambda} \in \mathbf{H}^{2}(\Omega)$ and $\partial_{t} \mathbf{F}_{\lambda} \in \mathbf{L}^{2}\left(Q_{T}\right)$, thanks to Lemma 5.3 and Eq. (5.5), we arrive at $\left\|\partial_{t} \mathbf{U}_{\lambda}\right\|_{\mathbf{L}^{2}\left(Q_{T}\right)} \leq C$. Thus, $\partial_{t} \mathbf{U}_{\lambda}$ weakly converges in $\mathbf{L}^{2}\left(Q_{T}\right)$ to some limit $\mathbf{W} \in \mathbf{L}^{2}\left(Q_{T}\right)$, which must be equal to $\partial_{t} \mathbf{U}$ by uniqueness of the limit in the distributional sense. This proves that $\partial_{t} \mathbf{U} \in \mathbf{L}^{2}\left(Q_{T}\right)$.

Additionally, due to (5.1), we find that

$$
\sup _{\lambda}\left(\lambda \int_{Q_{T}}\left(\mathcal{A} \mathbf{U}_{\lambda}\right) \cdot \mathbf{U}_{\lambda} \mathrm{d} x \mathrm{~d} t\right) \leq C,
$$

which implies that, in the limit, $u_{i}$ must be equal to zero a.e. in any set of the form $\left\{a_{i}>\varepsilon\right\}$ with $\varepsilon>0$. Indeed, for the union of sets, such that $n \in \mathbb{N}$ with $\varepsilon=1 / n$, we obtain that $u_{i}=0$ a.e. in $Q_{T} \backslash O_{a_{i}}$. Besides, $\mathbf{U}$ satisfies the system $\left(P_{\infty}\right)$ in the weak sense. Hence, let $\boldsymbol{\Phi}$ be a test function in $\mathbf{H}_{0}^{1}\left(Q_{T}\right)$, such that $\phi_{i}=0$ a.e. in $Q_{T} \backslash O_{a_{i}}$. The election of $\boldsymbol{\Phi}$ and the fact that $\mathbf{U}_{\lambda}$ is a solution of $\left(P_{\lambda}\right)$ implies

$$
\begin{gathered}
\int_{Q_{T}} \partial_{t} \mathbf{U}_{\lambda} \cdot \boldsymbol{\Phi} \mathrm{d} x \mathrm{~d} t+\int_{Q_{T}} D \mathbf{U}_{\lambda} \cdot D \boldsymbol{\Phi} \mathrm{d} x \mathrm{~d} t \\
-\int_{Q_{T}}\left(\mathcal{S} \mathbf{U}_{\lambda}\right) \cdot \boldsymbol{\Phi} \mathrm{d} x \mathrm{~d} t=\int_{Q_{T}} F_{\lambda} \cdot \boldsymbol{\Phi} \mathrm{d} x \mathrm{~d} t .
\end{gathered}
$$


Next, passing to the limit in the weak sense, we arrive at

$$
\int_{Q_{T}} \partial_{t} \mathbf{U} \cdot \boldsymbol{\Phi} \mathrm{d} x \mathrm{~d} t+\int_{Q_{T}} D \mathbf{U} \cdot D \boldsymbol{\Phi} \mathrm{d} x \mathrm{~d} t=\int_{Q_{T}} \mathbf{F} \cdot \boldsymbol{\Phi} \mathrm{d} x \mathrm{~d} t .
$$

Also, we observe that

$$
\int_{Q_{T}}(\mathcal{S U}) \cdot \boldsymbol{\Phi} \mathrm{d} x \mathrm{~d} t=0,
$$

since $\int_{Q_{T}} \alpha_{1} u_{2} \phi_{1} \mathrm{~d} x \mathrm{~d} t=0$ and $\int_{Q_{T}} \alpha_{2} u_{1} \phi_{2} \mathrm{~d} x \mathrm{~d} t=0$. Each term $u_{i, \lambda}$ mainly lives in $O_{a_{i}}$. Consequently, the coupling terms vanish as the value of $\lambda$ increases.

It remains to show that $\mathbf{U}$ is a solution of $\left(P_{\infty}\right)$. For that purpose, we prove that $\mathbf{U}(x, 0)=\mathbf{U}_{0}(x)$ a.e. in $x \in \Omega$. Then, let $\boldsymbol{\Phi} \in \mathbf{H}^{1}\left(Q_{T}\right)$ be any function that satisfies $\boldsymbol{\Phi}(T)=(0,0)^{T}$. Then, considering $\mathbf{U}_{\lambda}(0)=\mathbf{U}_{0, \lambda}$ in the weak formulation of $\left(P_{\lambda}\right)$, and integrating by parts, we get

$$
\begin{aligned}
& -\int_{\Omega} \mathbf{U}_{0, \lambda} \cdot \boldsymbol{\Phi}(0) \mathrm{d} x-\int_{Q_{T}} \mathbf{U}_{\lambda} \cdot \partial_{t} \mathbf{\Phi} \mathrm{d} x \mathrm{~d} t+\int_{Q_{T}} D \mathbf{U}_{\lambda} \cdot D \boldsymbol{\Phi} \mathrm{d} x \mathrm{~d} t \\
& =\int_{Q_{T}} \mathbf{F}_{\lambda} \cdot \boldsymbol{\Phi} \mathrm{d} x \mathrm{~d} t+\int_{Q_{T}}\left(\mathcal{S} \mathbf{U}_{\lambda}\right) \cdot \boldsymbol{\Phi} \mathrm{d} x \mathrm{~d} t .
\end{aligned}
$$

Passing to the limit as $\lambda$ tends to infinity and due to the weak convergence of $\mathbf{U}_{0, \lambda}$ to $\mathbf{U}_{0}$, we get

$$
\begin{aligned}
& -\int_{\Omega} \mathbf{U}_{0} \cdot \boldsymbol{\Phi}(0) \mathrm{d} x-\int_{Q_{T}} \mathbf{U} \cdot \partial_{t} \boldsymbol{\Phi} \mathrm{d} x \mathrm{~d} t \\
& \quad+\int_{Q_{T}} D \mathbf{U} \cdot D \boldsymbol{\Phi} \mathrm{d} x \mathrm{~d} t=\int_{Q_{T}} \mathbf{F} \cdot \boldsymbol{\Phi} \mathrm{d} x \mathrm{~d} t .
\end{aligned}
$$

Now, integrating by parts again, we can conclude that

$$
\begin{aligned}
& -\int_{\Omega} \mathbf{U}(0) \cdot \boldsymbol{\Phi}(0) \mathrm{d} x-\int_{Q_{T}} \mathbf{U} \cdot \partial_{t} \boldsymbol{\Phi} \mathrm{d} x \mathrm{~d} t \\
& \quad+\int_{Q_{T}} D \mathbf{U} \cdot D \boldsymbol{\Phi} \mathrm{d} x \mathrm{~d} t=\int_{Q_{T}} \mathbf{F} \cdot \boldsymbol{\Phi} \mathrm{d} x \mathrm{~d} t .
\end{aligned}
$$

Comparing Eqs. (5.8) and (5.9), we find that

$$
\int_{\Omega} \mathbf{U}_{0} \cdot \boldsymbol{\Phi}(0) \mathrm{d} x=\int_{\Omega} \mathbf{U}(0) \cdot \boldsymbol{\Phi}(0) \mathrm{d} x ;
$$

due to the fact that $\boldsymbol{\Phi}(0)$ is arbitrary, we deduce that $\mathbf{U}(0)=\mathbf{U}_{0}$ in $\mathbf{L}^{2}(\Omega)$.

Finally, the convergence of $\mathbf{U}_{\lambda}$ to $\mathbf{U}$ in $\mathbf{H}_{0}^{1}\left(Q_{T}\right)$ is satisfied a priori for some convergent subsequence. However, by uniqueness of the solution of problem $\left(P_{\infty}\right)$, see Proposition 4.1, the convergence is fulfilled for the whole sequence.

Corollary 5.5. Let $O_{a_{i}} \subset Q_{T}$ be time increasing open sets, such that potentials $a_{i}$ satisfy a time decay condition in $Q_{T}$ and let $\partial_{t} \mathbf{F}_{\lambda} \in \mathbf{L}^{2}\left(Q_{T}\right)$, $\mathbf{U}_{0, \lambda} \in \mathbf{H}^{2}(\Omega)$. Then, there exists a unique solution for the problem $\left(P_{\infty}\right)$.

Proof. It is sufficient to apply Proposition 5.4 with the following potentials $a_{i}(x, t)$ given by $\operatorname{dist}\left((x, t), \overline{O_{a_{i}}}\right)$, and such that $\mathbf{F}_{\lambda}=\mathbf{F}, \mathbf{U}_{0, \lambda}=\mathbf{U}_{0}$. 


\subsection{Strong Convergence in $\mathbf{H}_{0}^{1}\left(Q_{T}\right)$}

We use a similar argument to the one introduced in Sect. 2 to prove the strong convergence of the solutions $\mathbf{U}_{\lambda}$ of problem $\left(P_{\lambda}\right)$ to the solution $\mathbf{U}$ of problem $\left(P_{\infty}\right)$, when the parameter $\lambda$ goes to infinity.

Theorem 5.6. Let $\mathbf{U}_{\lambda}$ the solution of $\left(P_{\lambda}\right)$. Under the same hypothesis as in Proposition 5.4, $\mathbf{U}_{\lambda}$ converges in the strong topology of $\mathbf{H}_{0}^{1}\left(Q_{T}\right)$ to $\mathbf{U}$, solution of the problem $\left(P_{\infty}\right)$.

Proof. Lemma 5.1 shows that sequence $\left\{\mathbf{U}_{\lambda}\right\}$ is bounded in $\mathbf{H}_{0}^{1}\left(Q_{T}\right)$. On the other hand, Proposition 5.4 shows that $\mathbf{U}_{\lambda}$ weakly converges in $\mathbf{H}_{0}^{1}\left(Q_{T}\right)$ to $\mathbf{U}$, the unique solution of $\left(P_{\infty}\right)$. Moreover, the lower semicontinuity of the norm respect to the weak convergence implies

$$
\left\|\mathbf{U}_{\mathbf{H}_{0}^{1}\left(Q_{T}\right)} \leq \lim _{\lambda \rightarrow \infty} \inf \right\| \mathbf{U}_{\lambda} \|_{\mathbf{H}_{0}^{1}\left(Q_{T}\right)} .
$$

Thus, to obtain the strong convergence, we only have to prove the inequality involving the limit-sup. To do so observe that, for a.e. fixed $t, \mathbf{U}_{\lambda}$ solves $\mathcal{L} \mathbf{U}_{\lambda}+\lambda \mathcal{A} \mathbf{U}_{\lambda}=\mathbf{F}_{\lambda}+\mathcal{S} \mathbf{U}_{\lambda}-\partial_{t} \mathbf{U}_{\lambda}$, such that $\mathbf{U}_{\lambda}$ is a minimiser in $\mathbf{H}_{0}^{1}(\Omega)$ for the energy, that is

$$
\mathbf{W} \mapsto E_{\lambda}(\mathbf{W})-2 \int_{\Omega} \mathbf{F}_{\lambda} \cdot \mathbf{W} \mathrm{d} x-2 \int_{\Omega}\left(\mathcal{S} \mathbf{U}_{\lambda}\right) \cdot \mathbf{W} \mathrm{d} x+2 \int_{\Omega} \partial_{t} \mathbf{U}_{\lambda} \cdot \mathbf{W} \mathrm{d} x,
$$

where $E_{\lambda}$ is defined by (2.2). Furthermore, in Proposition 5.4 we obtained that

$$
\partial_{t} \mathbf{U}_{\lambda} \rightarrow \partial_{t} \mathbf{U} \text { weakly in } \mathbf{L}^{2}\left(Q_{T}\right) .
$$

Now, since $\left\|D \mathbf{U}_{\lambda}\right\|_{\mathbf{L}^{2}\left(Q_{T}\right)} \leq E_{\lambda}\left(\mathbf{U}_{\lambda}\right)$ for a.e. fixed $t$, we get

$$
\begin{aligned}
& \int_{\Omega}\left|D \mathbf{U}_{\lambda}\right|^{2} \mathrm{~d} x-2 \int_{\Omega}\left(\mathbf{F}_{\lambda}-\partial_{t} \mathbf{U}_{\lambda}\right) \cdot \mathbf{U}_{\lambda} \mathrm{d} x-2 \int_{\Omega}\left(\mathcal{S} \mathbf{U}_{\lambda}\right) \cdot \mathbf{U}_{\lambda} \mathrm{d} x \\
& \quad \leq E_{\lambda}\left(\mathbf{U}_{\lambda}\right)-2 \int_{\Omega}\left(\mathbf{F}_{\lambda}-\partial_{t} \mathbf{U}_{\lambda}\right) \cdot \mathbf{U}_{\lambda} \mathrm{d} x-2 \int_{\Omega}\left(\mathcal{S} \mathbf{U}_{\lambda}\right) \cdot \mathbf{U}_{\lambda} \mathrm{d} x \\
& \quad \leq E_{\lambda}(\mathbf{U})-2 \int_{\Omega}\left(\mathbf{F}_{\lambda}-\partial_{t} \mathbf{U}_{\lambda}\right) \cdot \mathbf{U} \mathrm{d} x \\
& \quad=\int_{\Omega}|D \mathbf{U}|^{2} \mathrm{~d} x-2 \int_{\Omega}\left(\mathbf{F}_{\lambda}-\partial_{t} \mathbf{U}_{\lambda}\right) \cdot \mathbf{U} \mathrm{d} x .
\end{aligned}
$$

Now, we integrate in $t \in[0, T]$ and consider the following: estimations

$$
\begin{gathered}
\int_{Q_{T}}\left(\mathbf{F}_{\lambda}-\partial_{t} \mathbf{U}_{\lambda}\right) \cdot \mathbf{U}_{\lambda} \mathrm{d} x \mathrm{~d} t \rightarrow \int_{Q_{T}}\left(\mathbf{F}-\partial_{t} \mathbf{U}\right) \cdot \mathbf{U} \mathrm{d} x \mathrm{~d} t, \\
\int_{Q_{T}}\left(\mathcal{S} \mathbf{U}_{\lambda}\right) \cdot \mathbf{U}_{\lambda} \mathrm{d} x \mathrm{~d} t \rightarrow 0 .
\end{gathered}
$$

Finally, calculating the limit-sup when $\lambda$ goes to infinity in (5.11) and using (5.12), we find that

$$
\|D \mathbf{U}\|_{\mathbf{L}^{2}\left(Q_{T}\right)} \geq \lim _{\lambda} \sup \left\|D \mathbf{U}_{\lambda}\right\|_{\mathbf{L}^{2}\left(Q_{T}\right)} .
$$

We know that every sequence $\mathbf{U}_{\lambda}$ converges to $\mathbf{U}$, solution of problem $\left(P_{\infty}\right)$, in the strong topology of $\mathbf{L}^{2}\left(Q_{T}\right)$, due to the fact that $\mathbf{U}_{\lambda}$ is bounded in 
$\mathbf{H}_{0}^{1}\left(Q_{T}\right)$ and the uniqueness of the solution of $\left(P_{\infty}\right)$. Thanks to inequalities (5.10), (5.13) and of the previous property, we conclude that every sequence $\mathbf{U}_{\lambda}$ converges in the strong topology of $\mathbf{H}_{0}^{1}\left(Q_{T}\right)$ to $\mathbf{U}$.

\section{Simon Exponential Estimate}

\subsection{Stationary Case}

As part of his Semiclassical works, Barry Simon, see [20, Theorem 4.1], established a strong convergence of the solutions for a class of Shrödinger operators of the form $\left(-h^{2} \Delta+V\right)$ in $\mathbb{R}^{N}$, where $V \geq 0$ is a $C^{\infty}$ potential bounded away from zero at infinity and having a finite number of non-degenerate zeros, multiple wells. In particular, it shows how the solutions for these operators decay exponentially as the so-called Planck's constant $h$ goes to zero in the regions where the potential is away from zero.

Following similar ideas, we obtain a very particular strong convergence of $\mathbf{U}_{\lambda}$, as stationary solutions of problem $\left(P_{\lambda}^{s}\right)$ far from the set $\Omega_{a_{1}} \cup \Omega_{a_{2}}$, see (2.1). To this aim, let $\varepsilon>0$ be fixed and define $\Omega_{\varepsilon, a_{i}}:=\left\{x \in \Omega\right.$; $\operatorname{dist}\left(x, \Omega_{a_{i}}\right)$ $>\varepsilon\}$, and

$$
\delta:=\min \left(\delta_{a_{1}}, \delta_{a_{2}}\right) \quad \text { with } \quad \delta_{a_{i}}:=\min _{x \in \bar{\Omega}_{\varepsilon, a_{i}}} a_{i}(x)>0 .
$$

Define also $\mathbf{H}=\left(\eta_{1}, \eta_{2}\right)^{T}$, where $\eta_{i}: \Omega \rightarrow \mathbb{R}$ satisfies $\eta_{i} \in W^{2, \infty}(\Omega)$ and is equal to 1 in $\Omega_{2 \varepsilon, a_{i}}$ and equal to 0 outside $\Omega_{\varepsilon, a_{i}}$. In the setting of Sect. 2, we prove the following estimate.

Lemma 6.1. Let $\mathbf{U}_{\lambda}$ the unique weak solution in $\mathbf{H}_{0}^{1}(\Omega)$ of the problem $\left(P_{\lambda}^{s}\right)$. There exists a constant $C>0$, such that for every $\lambda>0$

$$
\begin{gathered}
\int_{\Omega_{\varepsilon, a_{i}}} e^{2 \sqrt{\lambda \frac{\delta}{2}} \min \left(\operatorname{dist}\left(x, \Omega_{2 \varepsilon, a_{1}}^{c}\right), \operatorname{dist}\left(x, \Omega_{2 \varepsilon, a_{2}}^{c}\right)\right)} \eta_{i}^{2} u_{i, \lambda} \\
\left(\frac{\lambda \delta}{2} u_{i, \lambda}-f_{i, \lambda}-\alpha_{i} \widehat{u}_{i, \lambda}\right) \mathrm{d} x \leq C
\end{gathered}
$$

where $\widehat{u}_{1, \lambda}:=u_{2, \lambda}, \widehat{u}_{2, \lambda}:=u_{1, \lambda}$, and $C=C\left(\left\|\nabla \eta_{i}\right\|_{L^{\infty}(\Omega)},\left\|\Delta \eta_{i}\right\|_{L^{\infty}(\Omega)}, \varepsilon\right)$.

Proof. Let $\varepsilon>0$ be fixed. For any function $\Psi \in H_{0}^{1}\left(\Omega_{\varepsilon, a_{1}}\right) \times H_{0}^{1}\left(\Omega_{\varepsilon, a_{2}}\right)$ and any Lipschitz function $\rho$ that satisfies $|\nabla \rho|^{2} \leq \delta / 2$, we can calculate

$$
\begin{aligned}
\int_{\Omega} & \nabla\left(e^{\sqrt{\lambda} \rho} \psi_{i}\right) \cdot \nabla\left(e^{-\sqrt{\lambda} \rho} \psi_{i}\right) \mathrm{d} x \\
& =\int_{\Omega}\left(\sqrt{\lambda} e^{\sqrt{\lambda} \rho} \psi_{i} \nabla \rho+e^{\sqrt{\lambda} \rho} \nabla \psi_{i}\right) \cdot\left(-\sqrt{\lambda} e^{-\sqrt{\lambda} \rho} \psi_{i} \nabla \rho+e^{-\sqrt{\lambda} \rho} \nabla \psi_{i}\right) \mathrm{d} x \\
& =\int_{\Omega}\left(-\lambda \psi_{i}^{2}|\nabla \rho|^{2}+\left|\nabla \psi_{i}\right|^{2}\right) \mathrm{d} x,
\end{aligned}
$$

which impies

$$
\begin{gathered}
\int_{\Omega}\left(\nabla\left(e^{\sqrt{\lambda} \rho} \psi_{i}\right) \cdot \nabla\left(e^{-\sqrt{\lambda} \rho} \psi_{i}\right)+\lambda a_{i} \psi_{i}^{2}\right) \mathrm{d} x \\
\geq \int_{\Omega} \lambda\left(a_{i}-|\nabla \rho|^{2}\right) \psi_{i}^{2} \mathrm{~d} x \geq \frac{\lambda \delta}{2} \int_{\Omega} \psi_{i}^{2} \mathrm{~d} x
\end{gathered}
$$


due to the definition of $\delta$, see (6.1). If we choose $\boldsymbol{\Psi}=e^{\sqrt{\lambda} \rho} \mathbf{H} \cdot \mathbf{U}_{\lambda}$, the next computation holds

$$
\begin{aligned}
\nabla\left(e^{2 \sqrt{\lambda} \rho} \eta_{i}^{2} u_{i, \lambda}\right) \cdot \nabla u_{i, \lambda}= & {\left[\nabla\left(e^{2 \sqrt{\lambda} \rho} \eta_{i} u_{i, \lambda}\right)+e^{2 \sqrt{\lambda} \rho} u_{i, \lambda} \nabla \eta_{i}\right] \cdot \eta_{i} \nabla u_{i, \lambda} } \\
= & \nabla\left(e^{2 \sqrt{\lambda} \rho} \eta_{i} u_{i, \lambda}\right) \cdot\left(\nabla\left(\eta_{i} u_{i, \lambda}\right)-u_{i, \lambda} \nabla \eta_{i}\right) \\
& +e^{2 \sqrt{\lambda} \rho} u_{i, \lambda} \eta_{i} \nabla \eta_{i} \cdot \nabla u_{i, \lambda} .
\end{aligned}
$$

Since we have that $\nabla \eta_{i}=0$ in $\Omega_{2 \varepsilon, a_{i}}$ and in $\Omega_{\varepsilon, a_{i}}^{c}$, we arrive at the following expression on the left-hand side of inequality $(6.2)$ :

$$
\begin{aligned}
\int_{\Omega} & \left(\nabla\left(e^{\sqrt{\lambda} \rho} \psi_{i}\right) \cdot \nabla\left(e^{-\sqrt{\lambda} \rho} \psi_{i}\right)+\lambda a_{i} \psi_{i}^{2}\right) \mathrm{d} x \\
= & \int_{\Omega_{\varepsilon, a_{i}}}\left(\nabla\left(e^{2 \sqrt{\lambda} \rho} \eta_{i} u_{i, \lambda}\right) \cdot \nabla\left(\eta_{i} u_{i, \lambda}\right)+\lambda a_{i} e^{2 \sqrt{\lambda} \rho} \eta_{i}^{2} u_{i, \lambda}^{2}\right) \mathrm{d} x \\
= & \int_{\Omega_{\varepsilon, a_{i}}}\left(\nabla\left(e^{2 \sqrt{\lambda} \rho} \eta_{i}^{2} u_{i, \lambda}\right) \cdot \nabla u_{i, \lambda}+\lambda a_{i} e^{2 \sqrt{\lambda} \rho} \eta_{i}^{2} u_{i, \lambda}^{2}\right) \mathrm{d} x \\
& -\int_{\Omega_{\varepsilon, a_{i}} \backslash \overline{\Omega_{2 \varepsilon, a_{i}}}} e^{2 \sqrt{\lambda} \rho} u_{i, \lambda} \eta_{i} \nabla \eta_{i} \cdot \nabla u_{i, \lambda} \mathrm{d} x \\
& +\int_{\Omega_{\varepsilon, a_{i}} \backslash \overline{\Omega_{2 \varepsilon, a_{i}}}} \nabla\left(e^{2 \sqrt{\lambda} \rho} \eta_{i} u_{i, \lambda}\right) \cdot u_{i, \lambda} \nabla \eta_{i} \mathrm{~d} x .
\end{aligned}
$$

Thus, $e^{2 \sqrt{\lambda} \rho} \eta_{i}^{2} u_{i, \lambda} \in H_{0}^{1}\left(\Omega_{\varepsilon, a_{i}}\right)$ provides us with an admissible test function for problem $\left(P_{\lambda}^{s}\right)$ and we find that

$$
\begin{aligned}
& \int_{\Omega_{\varepsilon, a_{i}}}\left(\nabla\left(e^{2 \sqrt{\lambda} \rho} \eta_{i}^{2} u_{i, \lambda}\right) \cdot \nabla u_{i, \lambda}+\lambda a_{i} e^{2 \sqrt{\lambda} \rho} \eta_{i}^{2} u_{i, \lambda}^{2}-\alpha_{i} e^{2 \sqrt{\lambda} \rho} \eta_{i}^{2} u_{i, \lambda} \widehat{u}_{i, \lambda}\right) \mathrm{d} x \\
& \quad=\int_{\Omega_{\varepsilon, a_{i}}} e^{2 \sqrt{\lambda} \rho} \eta_{i}^{2} u_{i, \lambda} f_{i, \lambda} \mathrm{d} x
\end{aligned}
$$

where $\widehat{u}_{1, \lambda} \equiv u_{2, \lambda}, \widehat{u}_{2, \lambda} \equiv u_{1, \lambda}$. Moreover, Proposition 2.3 implies

$$
\left\|\mathbf{U}_{\lambda}\right\|_{\mathbf{L}^{2}(\Omega)}+\left\|D \mathbf{U}_{\lambda}\right\|_{\mathbf{L}^{2}(\Omega)} \leq C\left\|\mathbf{F}_{\lambda}\right\|_{\mathbf{L}^{2}(\Omega)},
$$

for a constant $C$. In addition, Hölder's inequality allows us to obtain

$$
\left|\int_{\Omega_{\varepsilon, a_{i}} \backslash \overline{\Omega_{2 \varepsilon, a_{i}}}} e^{2 \sqrt{\lambda} \rho} u_{i, \lambda} \eta_{i} \nabla \eta_{i} \cdot \nabla u_{i, \lambda} \mathrm{d} x\right| \leq C\left\|\nabla \eta_{i}\right\|_{L^{\infty}(\Omega)}\left\|f_{i, \lambda}\right\|_{L^{2}(\Omega)}^{2} e^{2 \sqrt{\lambda} m_{i}},
$$

where $m_{i}$ is defined by

$$
m_{i}:=\sup _{x \in \Omega_{\varepsilon, a_{i}} \backslash \overline{\Omega_{2 \varepsilon, a_{i}}}} \rho(x) .
$$

Due to the fact that $u_{i, \lambda} \nabla \eta_{i} \in H_{0}^{1}\left(\Omega_{\varepsilon, a_{i}} \backslash \overline{\Omega_{2 \varepsilon, a_{i}}}\right)$ and $\eta_{i} \in W^{2, \infty}(\Omega)$, we can integrate by parts the following term of (6.3) and get:

$$
\begin{aligned}
\int_{\Omega_{\varepsilon, a_{i}} \backslash \overline{\Omega_{2 \varepsilon, a_{i}}}} \nabla\left(e^{2 \sqrt{\lambda} \rho} \eta_{i} u_{i, \lambda}\right) \cdot\left(u_{i, \lambda} \nabla \eta_{i}\right) \mathrm{d} x= & -\int_{\Omega_{\varepsilon, a_{i}} \backslash \overline{\Omega_{2 \varepsilon, a_{i}}}} e^{2 \sqrt{\lambda} \rho} \eta_{i} u_{i, \lambda}\left(u_{i, \lambda} \Delta \eta_{i}\right. \\
& \left.+\nabla u_{i, \lambda} \cdot \nabla \eta_{i}\right) \mathrm{d} x .
\end{aligned}
$$


Consequently

$$
\begin{aligned}
\mid \int_{\Omega_{\varepsilon, a_{i}} \backslash \frac{\Omega_{2 \varepsilon, a_{i}}}{} \nabla\left(e^{2 \sqrt{\lambda} \rho} \eta_{i} u_{i, \lambda}\right) \cdot\left(u_{i, \lambda} \nabla \eta_{i}\right) \mathrm{d} x \mid \leq} & C\left(\left\|\nabla \eta_{i}\right\|_{L^{\infty}(\Omega)}\right. \\
& \left.+\left\|\Delta \eta_{i}\right\|_{L^{\infty}(\Omega)}\right)\left\|f_{i, \lambda}\right\|_{L^{2}(\Omega)}^{2} e^{2 \sqrt{\lambda} m_{i}} .
\end{aligned}
$$

Combining the estimations (6.3), (6.4), (6.5), and (6.6) with (6.2), we arrive at

$$
\begin{aligned}
\frac{\lambda \delta}{2} \int_{\Omega_{\varepsilon, a_{i}}} e^{2 \sqrt{\lambda} \rho} \eta_{i}^{2} u_{i, \lambda}^{2} \mathrm{~d} x \leq & \int_{\Omega_{\varepsilon, a_{i}}} e^{2 \sqrt{\lambda} \rho} \eta_{i}^{2} u_{i, \lambda} f_{i, \lambda} \mathrm{d} x \\
& +\int_{\Omega_{\varepsilon, a_{i}}} \alpha_{i} e^{2 \sqrt{\lambda} \rho} \eta_{i}^{2} u_{i, \lambda} \widehat{u}_{i, \lambda} \mathrm{d} x+C e^{2 \sqrt{\lambda} m_{i}},
\end{aligned}
$$

where $C=C\left(\left\|\nabla \eta_{i}\right\|_{L^{\infty}(\Omega)},\left\|\Delta \eta_{i}\right\|_{L^{\infty}(\Omega)}, \varepsilon\right)$. In particular, we specify the function $\rho$ by setting

$$
\rho(x):=\sqrt{\frac{\delta}{2}} \min \left(\operatorname{dist}\left(x, \Omega_{2 \varepsilon, a_{1}}^{c}\right), \operatorname{dist}\left(x, \Omega_{2 \varepsilon, a_{2}}^{c}\right)\right),
$$

which satisfies all our needed assumptions ( $\rho$ is Lipschitz, $|\nabla \rho|^{2} \leq \delta / 2$ and $\rho=0$ outside $\left.\Omega_{\varepsilon, a_{1}} \cup \Omega_{\varepsilon, a_{2}}\right)$. In this case, $m_{i}=0$ which implies that Eq. (6.7) becomes

$$
\begin{aligned}
& \frac{\lambda \delta}{2} \int_{\Omega_{\varepsilon, a_{i}}} e^{2 \sqrt{\lambda} \rho} \eta_{i}^{2} u_{i, \lambda}^{2} \mathrm{~d} x \leq \int_{\Omega_{\varepsilon, a_{i}}} e^{2 \sqrt{\lambda} \rho} \eta_{i}^{2} u_{i, \lambda} f_{i, \lambda} \mathrm{d} x \\
& \quad+\int_{\Omega_{\varepsilon, a_{i}}} \alpha_{i} e^{2 \sqrt{\lambda} \rho} \eta_{i}^{2} u_{i, \lambda} \widehat{u}_{i, \lambda} \mathrm{d} x+C,
\end{aligned}
$$

or equivalently

$$
\int_{\Omega_{\varepsilon, a_{i}}} e^{2 \sqrt{\lambda} \rho} \eta_{i}^{2} u_{i, \lambda}\left(\frac{\lambda \delta}{2} u_{i, \lambda}-f_{i, \lambda}-\alpha_{i} \widehat{u}_{i, \lambda}\right) \mathrm{d} x \leq C,
$$

which completes the proof.

Remark 6.1. We can use the previous lemma when $f_{i}=0$ in $\Omega \backslash \Omega_{a_{i}}$. Thus, the convergence ratio $\mathbf{U}_{\lambda} \rightarrow 0$ when $\lambda \rightarrow \infty$ far from $\Omega_{a_{1}} \cup \Omega_{a_{2}}$ is

$$
\int_{\Omega_{2 \varepsilon, a_{i}}} e^{2 \sqrt{\lambda \frac{\delta}{2}} \min \left(\operatorname{dist}\left(x, \Omega_{2 \varepsilon, a_{1}}^{c}\right), \operatorname{dist}\left(x, \Omega_{2 \varepsilon, a_{2}}^{c}\right)\right)} u_{i, \lambda}\left(\frac{\lambda \delta}{2} u_{i, \lambda}-\alpha_{i} \widehat{u}_{i, \lambda}\right) \mathrm{d} x \leq C .
$$

This result considerately improves the previously mentioned estimation

$$
\lambda \int_{\Omega}\left(\mathcal{A} \mathbf{U}_{\lambda}\right) \cdot \mathbf{U}_{\lambda} \mathrm{d} x \leq C .
$$




\subsection{Parabolic Case}

We extend the previous bound corresponding to the stationary case for the parabolic case. To this aim, similarly as before, for each $\varepsilon>0$, we define

$$
\delta_{a_{i}}:=\min _{(x, t) \in \overline{A_{\varepsilon, a_{i}}}} a_{i}(x, t)>0, \quad \delta=\min \left(\delta_{a_{1}}, \delta_{a_{2}}\right) .
$$

with $A_{\varepsilon, a_{i}}:=\left\{(x, t) \in \overline{Q_{T}} ; \operatorname{dist}\left((x, t), O_{a_{i}}\right)>\varepsilon\right\}$. We also consider the function $\mathbf{H}=\left(\eta_{1}, \eta_{2}\right)^{T}, \eta_{i}: Q_{T} \rightarrow \mathbb{R}$, such that $\eta_{i} \in W^{2, \infty}\left(Q_{T}\right)$, is equal to 1 in $A_{2 \varepsilon, a_{i}}$ and equal to 0 outside $A_{\varepsilon, a_{i}}$.

Lemma 6.2. Let $\mathbf{U}_{\lambda}$ be the solution of problem $\left(P_{\lambda}\right)$ with initial data $\mathbf{U}_{0, \lambda} \in$ $H_{0}^{1}\left(\overline{O_{a_{1}}} \cap\{t=0\}\right) \times H_{0}^{1}\left(\overline{O_{a_{2}}} \cap\{t=0\}\right)$.

Then, for each $\lambda \geq 4$, there exists a constant $C>0$, such that

$$
\begin{gathered}
\int_{A_{\varepsilon, a_{i}}} e^{2 \sqrt{\lambda} c_{\delta} \min \left(\operatorname{dist}\left((x, t), A_{2 \varepsilon, a_{1}}^{c}\right), \operatorname{dist}\left((x, t), A_{2 \varepsilon, a_{2}}^{c}\right)\right)} \eta_{i}^{2} u_{i, \lambda} \\
\left(\frac{\lambda \delta}{4} u_{i, \lambda}-f_{i, \lambda}-\alpha_{i} \widehat{u}_{i, \lambda}\right) \mathrm{d} x \mathrm{~d} t \leq C
\end{gathered}
$$

where $\widehat{u}_{1, \lambda}:=u_{2, \lambda}, \widehat{u}_{2, \lambda}:=u_{1, \lambda}, c_{\delta}:=\min \left(\sqrt{\frac{\delta}{2}}, \frac{\delta}{2}\right)$, and the constant $C$ depends on $\varepsilon,\left\|\nabla \eta_{i}\right\|_{L^{\infty}\left(Q_{T}\right)},\left\|\Delta \eta_{i}\right\|_{L^{\infty}\left(Q_{T}\right)}$ and $\left\|\partial_{t} \eta_{i}\right\|_{L^{\infty}\left(Q_{T}\right)}$.

Proof. Let $\varepsilon>0$ be a fixed value. Consider any function $\boldsymbol{\Psi} \in \mathbf{H}^{1}\left(Q_{T}\right)$ that satisfies $\psi_{i}=0$ a.e. in $A_{\varepsilon, a_{i}}^{c}$ and any Lipschitz function $\rho: Q_{T} \rightarrow \mathbb{R}$ that fulfils

$$
\max \left(\left|\partial_{t} \rho\right|,|\nabla \rho|^{2}\right) \leq \delta / 2
$$

Integrating in time the estimate (6.2) and using the definition of $\delta$ given by (6.8), we obtain

$$
\int_{Q_{T}}\left(\nabla\left(e^{\sqrt{\lambda} \rho} \psi_{i}\right) \cdot \nabla\left(e^{-\sqrt{\lambda} \rho} \psi_{i}\right)+\lambda a_{i} \psi_{i}^{2}\right) \mathrm{d} x \mathrm{~d} t \geq \frac{\lambda \delta}{2} \int_{Q_{T}} \psi_{i}^{2} \mathrm{~d} x \mathrm{~d} t .(6.1
$$

We focus now on the time derivative and use estimate (6.9) to get the following expression:

$$
\begin{aligned}
\int_{Q_{T}} e^{\sqrt{\lambda} \rho} \psi_{i} \partial_{t}\left(e^{-\sqrt{\lambda} \rho} \psi_{i}\right) \mathrm{d} x \mathrm{~d} t= & -\sqrt{\lambda} \int_{Q_{T}} \psi_{i}^{2} \partial_{t} \rho \mathrm{d} x \mathrm{~d} t+\int_{Q_{T}} \psi_{i} \partial_{t} \psi_{i} \mathrm{~d} x \mathrm{~d} t \\
= & -\sqrt{\lambda} \int_{Q_{T}} \psi_{i}^{2} \partial_{t} \rho \mathrm{d} x \mathrm{~d} t \\
& +\frac{1}{2}\left(\int_{\Omega} \psi_{i}(T)^{2} \mathrm{~d} x-\int_{\Omega} \psi_{i}(0)^{2} \mathrm{~d} x\right) \\
\geq & -\frac{\sqrt{\lambda} \delta}{2} \int_{Q_{T}} \psi_{i}^{2} \mathrm{~d} x \mathrm{~d} t-\frac{1}{2} \int_{\Omega} \psi_{i}(0)^{2} \mathrm{~d} x
\end{aligned}
$$


Estimates (6.10), (6.11) allow us to obtain

$$
\begin{aligned}
& \int_{Q_{T}}\left(e^{\sqrt{\lambda} \rho} \psi_{i}\left(\partial_{t}\left(e^{-\sqrt{\lambda} \rho} \psi_{i}\right)+\lambda a_{i} e^{-\sqrt{\lambda} \rho} \psi_{i}\right)+\nabla\left(e^{\sqrt{\lambda} \rho} \psi_{i}\right) \cdot \nabla\left(e^{-\sqrt{\lambda} \rho} \psi_{i}\right)\right) \mathrm{d} x \mathrm{~d} t \\
& \quad \geq \frac{\delta}{2}(\lambda-\sqrt{\lambda}) \int_{Q_{T}} \psi_{i}^{2} \mathrm{~d} x \mathrm{~d} t-\frac{1}{2} \int_{\Omega} \psi_{i}(0)^{2} \mathrm{~d} x
\end{aligned}
$$

Moreover, choose $\psi=e^{\sqrt{\lambda} \rho} \mathbf{H} \cdot \mathbf{U}_{\lambda}$. Assume also that $\lambda \geq 4$, so that $\lambda-\sqrt{\lambda} \geq$ $\lambda / 2$ is accomplished. Since $\mathbf{U}_{0, \lambda} \in H_{0}^{1}\left(\overline{O_{a_{1}}} \cap\{t=0\}\right) \times \bar{H}_{0}^{1}\left(\overline{O_{a_{2}}} \cap\{t=0\}\right)$, we have that $\left\|\psi_{i}(0)\right\|_{L^{2}(\Omega)}=0$. Hence, we can rewrite equation (6.12) as follows:

$$
\begin{aligned}
& \frac{\lambda \delta}{4} \int_{A_{\varepsilon, a_{i}}} e^{2 \sqrt{\lambda} \rho} \eta_{i}^{2} u_{i, \lambda}^{2} \mathrm{~d} x \mathrm{~d} t \\
& \leq \int_{A_{\varepsilon, a_{i}}}\left(\nabla\left(e^{2 \sqrt{\lambda} \rho} \eta_{i} u_{i, \lambda}\right) \cdot \nabla\left(\eta_{i} u_{i, \lambda}\right)+\lambda a_{i} e^{2 \sqrt{\lambda} \rho} \eta_{i}^{2} u_{i, \lambda}^{2}\right. \\
& \left.\quad+e^{2 \sqrt{\lambda} \rho} \eta_{i} u_{i, \lambda} \partial_{t}\left(\eta_{i} u_{i, \lambda}\right)\right) \mathrm{d} x \mathrm{~d} t .
\end{aligned}
$$

Proceeding analogously as in the stationary case

$$
\begin{aligned}
& \int_{A_{\varepsilon, a_{i}}}\left(\nabla\left(e^{2 \sqrt{\lambda} \rho} \eta_{i} u_{i, \lambda}\right) \cdot \nabla\left(\eta_{i} u_{i, \lambda}\right)+\lambda a_{i} e^{2 \sqrt{\lambda} \rho} \eta_{i}^{2} u_{i, \lambda}^{2}\right) \mathrm{d} x \mathrm{~d} t \\
& =\int_{A_{\varepsilon, a_{i}}}\left(\nabla\left(e^{2 \sqrt{\lambda} \rho} \eta_{i}^{2} u_{i, \lambda}\right) \cdot \nabla u_{i, \lambda}+\lambda a_{i} e^{2 \sqrt{\lambda} \rho} \eta_{i}^{2} u_{i, \lambda}^{2}\right) \mathrm{d} x \mathrm{~d} t \\
& \quad-\int_{A_{\varepsilon, a_{i}} \backslash \overline{A_{2 \varepsilon, a_{i}}}} e^{2 \sqrt{\lambda} \rho} u_{i, \lambda} \eta_{i} \nabla \eta_{i} \cdot \nabla u_{i, \lambda} \mathrm{d} x \mathrm{~d} t \\
& +\int_{A_{\varepsilon, a_{i}} \backslash \overline{A_{2 \varepsilon, a_{i}}}} \nabla\left(e^{2 \sqrt{\lambda} \rho} \eta_{i} u_{i, \lambda}\right) \cdot u_{i, \lambda} \nabla \eta_{i} \mathrm{~d} x \mathrm{~d} t .
\end{aligned}
$$

We recall that according to bound (5.1), there exists a constant $C>0$, such that

$$
\left\|\mathbf{U}_{\lambda}\right\|_{\mathbf{H}^{1}\left(Q_{T}\right)} \leq C\left(\left\|\mathbf{F}_{\lambda}\right\|_{\mathbf{L}^{2}\left(Q_{T}\right)}+\left\|\mathbf{U}_{0, \lambda}\right\|_{\mathbf{L}^{2}(\Omega)}\right) .
$$

Thus, due to Hölder's inequality

$$
\begin{aligned}
& \left|\int_{A_{\varepsilon, a_{i}} \backslash \overline{A_{2 \varepsilon, a_{i}}}} e^{2 \sqrt{\lambda} \rho} u_{i, \lambda} \eta_{i} \nabla \eta_{i} \cdot \nabla u_{i, \lambda} \mathrm{d} x \mathrm{~d} t\right| \\
& \quad \leq C e^{2 \sqrt{\lambda} m_{i}}\left\|\nabla \eta_{i}\right\|_{L^{\infty}(\Omega)}\left(\left\|f_{i, \lambda}\right\|_{L^{2}\left(Q_{T}\right)}+\left\|u_{0 i, \lambda}\right\|_{L^{2}(\Omega)}\right)^{2}
\end{aligned}
$$

where $m_{i}$ is defined as

$$
m_{i}:=\sup _{(x, t) \in A_{\varepsilon, a_{i}} \backslash \overline{A_{2 \varepsilon, a_{i}}}} \rho(x, t) .
$$

Notice that $u_{i, \lambda} \nabla \eta_{i}$ cancels out outside $A_{\varepsilon, a_{i}} \backslash \overline{A_{2 \varepsilon, a_{i}}}$ and $\eta_{i} \in W^{2, \infty}\left(Q_{T}\right)$

$$
\begin{aligned}
& \int_{A_{\varepsilon, a_{i} \backslash} \backslash \overline{A_{2 \varepsilon, a_{i}}}} \nabla\left(e^{2 \sqrt{\lambda} \rho} \eta_{i} u_{i, \lambda}\right) \cdot u_{i, \lambda} \nabla \eta_{i} \mathrm{~d} x \mathrm{~d} t \\
& \quad=-\int_{A_{\varepsilon, a_{i}} \backslash \overline{A_{2 \varepsilon, a_{i}}}} e^{2 \sqrt{\lambda} \rho} \eta_{i} u_{i, \lambda}\left(u_{i, \lambda} \Delta \eta_{i}+\nabla u_{i, \lambda} \cdot \nabla \eta_{i}\right) \mathrm{d} x \mathrm{~d} t .
\end{aligned}
$$


Hence, integrating by parts on the spatial domain, we find that

$$
\begin{aligned}
& \left|\int_{A_{\varepsilon, a_{i}} \backslash \overline{A_{2 \varepsilon, a_{i}}}} \nabla\left(e^{2 \sqrt{\lambda} \rho} \eta_{i} u_{i, \lambda}\right) \cdot u_{i, \lambda} \nabla \eta_{i} \mathrm{~d} x \mathrm{~d} t\right| \\
& \quad \leq C e^{2 \sqrt{\lambda} m_{i}}\left(\left\|\nabla \eta_{i}\right\|_{L^{\infty}\left(Q_{T}\right)}+\left\|\Delta \eta_{i}\right\|_{L^{\infty}\left(Q_{T}\right)}\right)\left(\left\|f_{i, \lambda}\right\|_{L^{2}\left(Q_{T}\right)}+\left\|u_{0 i, \lambda}\right\|_{L^{2}(\Omega)}\right)^{2} .
\end{aligned}
$$

We decompose the term corresponding to the time derivative on the right hand side of inequality (6.13) in two terms

$$
\begin{aligned}
\int_{A_{\varepsilon, a_{i}}} e^{2 \sqrt{\lambda} \rho} \eta_{i} u_{i, \lambda} \partial_{t}\left(\eta_{i} u_{i, \lambda}\right) \mathrm{d} x \mathrm{~d} t= & \int_{A_{\varepsilon, a_{i}}} e^{2 \sqrt{\lambda} \rho} \eta_{i}^{2} u_{i, \lambda} \partial_{t} u_{i, \lambda} \mathrm{d} x \mathrm{~d} t \\
& +\int_{A_{\varepsilon, a_{i}}} e^{2 \sqrt{\lambda} \rho} \eta_{i} u_{i, \lambda}^{2} \partial_{t} \eta_{i} \mathrm{~d} x \mathrm{~d} t
\end{aligned}
$$

and due to the fact that $\partial_{t} \eta_{i}$ cancels out outside $A_{\varepsilon, a_{i}} \backslash \overline{A_{2 \varepsilon, a_{i}}}$, we find the upper bound

$$
\begin{aligned}
\left|\int_{A_{\varepsilon, a_{i}} \backslash \overline{A_{2 \varepsilon, a_{i}}}} e^{2 \sqrt{\lambda} \rho} \eta_{i} u_{i, \lambda}^{2} \partial_{t} \eta_{i} \mathrm{~d} x \mathrm{~d} t\right| \leq & C e^{2 \sqrt{\lambda} m_{i}}\left(\left\|\partial_{t} \eta_{i}\right\|_{L^{\infty}\left(Q_{T}\right)}\right)\left(\left\|f_{i, \lambda}\right\|_{L^{2}\left(Q_{T}\right)}\right. \\
& \left.+\left\|u_{0 i, \lambda}\right\|_{L^{2}(\Omega)}\right)^{2} .
\end{aligned}
$$

Consequently, we rewrite inequality (6.13) using (6.14)-(6.16)

$$
\begin{aligned}
& \frac{\lambda \delta}{4} \int_{A_{\varepsilon, a_{i}}} e^{2 \sqrt{\lambda} \rho} \eta_{i}^{2} u_{i, \lambda}^{2} \mathrm{~d} x \mathrm{~d} t \leq C e^{2 \sqrt{\lambda} m_{i}} \\
& \quad+\int_{A_{\varepsilon, a_{i}}}\left(\nabla\left(e^{2 \sqrt{\lambda} \rho} \eta_{i}^{2} u_{i, \lambda}\right) \cdot \nabla u_{i, \lambda}+\lambda a_{i} e^{2 \sqrt{\lambda} \rho} \eta_{i}^{2} u_{i, \lambda}^{2}+e^{2 \sqrt{\lambda} \rho} \eta_{i}^{2} u_{i, \lambda} \partial_{t} u_{i, \lambda}\right) \mathrm{d} x \mathrm{~d} t,
\end{aligned}
$$

where $C=C\left(\left\|\nabla \eta_{i}\right\|_{L^{\infty}(\Omega)},\left\|\Delta \eta_{i}\right\|_{L^{\infty}(\Omega)},\left\|\partial_{t} \eta_{i}\right\|_{L^{\infty}(\Omega)}, \varepsilon\right)$. Since $\eta_{i}$ is identically zero outside $A_{\varepsilon, a_{i}}$, the function $e^{2 \sqrt{\lambda} \rho} \eta_{i}^{2} u_{i, \lambda} \in L^{2}\left(0, T ; H_{0}^{1}(\Omega)\right)$ is an admissible test function for each component $i$ of $\left(P_{\lambda}\right)$. Therefore

$$
\begin{gathered}
\int_{A_{\varepsilon, a_{i}}}\left(\nabla\left(e^{2 \sqrt{\lambda} \rho} \eta_{i}^{2} u_{i, \lambda}\right) \cdot \nabla u_{i, \lambda}+\lambda a_{i} e^{2 \sqrt{\lambda} \rho} \eta_{i}^{2} u_{i, \lambda}^{2}+e^{2 \sqrt{\lambda} \rho} \eta_{i}^{2} u_{i, \lambda} \partial_{t} u_{i, \lambda}\right) \mathrm{d} x \mathrm{~d} t \\
=\int_{A_{\varepsilon, a_{i}}} e^{2 \sqrt{\lambda} \rho} \eta_{i}^{2} u_{i, \lambda} f_{i, \lambda} \mathrm{d} x \mathrm{~d} t+\int_{A_{\varepsilon, a_{i}}} \alpha_{i} e^{2 \sqrt{\lambda} \rho} \eta_{i}^{2} u_{i, \lambda} \widehat{u}_{i, \lambda} \mathrm{d} x \mathrm{~d} t,
\end{gathered}
$$

which implies that

$$
\begin{aligned}
& \frac{\lambda \delta}{4} \int_{A_{\varepsilon, a_{i}}} e^{2 \sqrt{\lambda} \rho} \eta_{i}^{2} u_{i, \lambda}^{2} \mathrm{~d} x \mathrm{~d} t \\
& \quad \leq C e^{2 \sqrt{\lambda} m_{i}}+\int_{A_{\varepsilon, a_{i}}} e^{2 \sqrt{\lambda} \rho} \eta_{i}^{2} u_{i, \lambda} f_{i, \lambda} \mathrm{d} x \mathrm{~d} t+\int_{A_{\varepsilon, a_{i}}} e^{2 \sqrt{\lambda} \rho} \eta_{i}^{2} \alpha_{i} u_{i, \lambda} \widehat{u}_{i, \lambda} \mathrm{d} x \mathrm{~d} t .
\end{aligned}
$$


Finally, we choose as a particular case

$$
\rho(x, t):=\min \left(\sqrt{\frac{\delta}{2}}, \frac{\delta}{2}\right) \min \left(\operatorname{dist}\left((x, t), A_{2 \varepsilon, a_{1}}^{c}\right), \operatorname{dist}\left((x, t), A_{2 \varepsilon, a_{2}}^{c}\right)\right),
$$

which satisfy all our needed conditions ( $\rho$ is Lipschitz, $\left|\partial_{t} \rho\right|+|\nabla \rho|^{2} \leq \delta / 2$ and has support on $\left.A_{\varepsilon, a_{1}} \cup A_{\varepsilon, a_{2}}\right)$. In this case, $m_{i}=0$, therefore, expression (6.17) is reduced to

$$
\begin{aligned}
& \frac{\lambda \delta}{4} \int_{A_{\varepsilon, a_{i}}} e^{2 \sqrt{\lambda} \rho} \eta_{i}^{2} u_{i, \lambda}^{2} \mathrm{~d} x \mathrm{~d} t \leq C+\int_{A_{\varepsilon, a_{i}}} e^{2 \sqrt{\lambda} \rho} \eta_{i}^{2} u_{i, \lambda} f_{i, \lambda} \mathrm{d} x \mathrm{~d} t \\
& \quad+\int_{A_{\varepsilon, a_{i}}} e^{2 \sqrt{\lambda} \rho} \eta_{i}^{2} \alpha_{i} u_{i, \lambda} \widehat{u}_{i, \lambda} \mathrm{d} x \mathrm{~d} t .
\end{aligned}
$$

Equivalently

$$
\int_{A_{\varepsilon, a_{i}}} e^{2 \sqrt{\lambda} \rho} \eta_{i}^{2} u_{i, \lambda}\left(\frac{\lambda \delta}{4} u_{i, \lambda}-f_{i, \lambda}-\alpha_{i} \widehat{u}_{i, \lambda}\right) \mathrm{d} x \mathrm{~d} t \leq C .
$$

To conclude, we observe that the previous lemma can be specified for a linear eigenvalue problem with $f_{i, \lambda}=\sigma u_{i, \lambda}$, where $\sigma$ stands for an eigenvalue associated with the eigenfunction $u_{i, \lambda}$. Then, thanks to Lemma 6.2, we find a decay estimation for the eigenfunctions on the boundary of the regions $O_{a_{i}}$.

Corollary 6.3. In the particular case where $f_{i}=0$ in $Q_{T} \backslash O_{a_{i}}$, we obtain the following estimation of the solutions' convergence $\mathbf{U}_{\lambda} \rightarrow \mathbf{0}$ of problem $\left(P_{\lambda}\right)$ when $\lambda \rightarrow \infty$ outside the regions $O_{a_{i}}$ :

$e^{2 c_{\delta} \varepsilon \sqrt{\lambda}} \int_{A_{2 \varepsilon, a_{i}}} u_{i, \lambda}\left(\frac{\lambda \delta}{4} u_{i, \lambda}-\alpha_{i} \widehat{u}_{i, \lambda}\right) \mathrm{d} x \mathrm{~d} t \leq C, \quad$ with $c_{\delta}:=\min \left(\sqrt{\frac{\delta}{2}}, \frac{\delta}{2}\right)$.

Funding Open Access funding provided thanks to the CRUE-CSIC agreement with Springer Nature.

Open Access. This article is licensed under a Creative Commons Attribution 4.0 International License, which permits use, sharing, adaptation, distribution and reproduction in any medium or format, as long as you give appropriate credit to the original author(s) and the source, provide a link to the Creative Commons licence, and indicate if changes were made. The images or other third party material in this article are included in the article's Creative Commons licence, unless indicated otherwise in a credit line to the material. If material is not included in the article's Creative Commons licence and your intended use is not permitted by statutory regulation or exceeds the permitted use, you will need to obtain permission directly from the copyright holder. To view a copy of this licence, visit http:// creativecommons.org/licenses/by/4.0/.

Publisher's Note Springer Nature remains neutral with regard to jurisdictional claims in published maps and institutional affiliations. 


\section{References}

[1] Álvarez-Caudevilla, P., López-Gómez, J.: Asymptotic behaviour of principal eigenvalues for a class of cooperative systems. J. Differ. Equations 244(5), 10931113 (2008) [Corrigendum in Journal of Differential Equations 245(2), 566-567 (2008)]

[2] Álvarez-Caudevilla, P., López-Gómez, J.: Semiclassical analysis for highly degenerate potentials. Proc. Am. Math. Soc. 136(2), 665-675 (2008)

[3] Álvarez-Caudevilla, P., Lemenant, A.: Asymptotic analysis for some linear eigenvalue problems via Gamma-convergence. Adv. Differ. Equations 15(7-8), 649-688 (2010)

[4] Álvarez-Caudevilla, P., Du, Y., Peng, R.: Qualitative analysis of a cooperative reaction-diffusion system in a spatiotemporally degenerate environment. SIAM J. Math. Anal. 46(1), 499-531 (2014)

[5] Amann, H.: Maximum Principle and Principal Eigenvalues. In: Ferrera, J., López-Gómez, J., Ruiz del Portal, F.R. (eds.) Ten Mathematical Essays on Approximation in Analysis and Topology, pp. 1-60. Elsevier, Amsterdam (2005)

[6] Antón, I., López-Gómez, J.: The strong maximum principle for cooperative periodic-parabolic systems and the existence of principal eigenvalues. World Congress of Nonlinear Analysts '92, Vol. I-IV (Tampa, FL, 1992), pp. 323334. de Gruyter, Berlin, (1996)

[7] Arnold, V.I.: Ordinary Differential Equations. Translated from the third Russian edition by Roger Cooke. Springer Textbook. Springer, Berlin (1992)

[8] Brézis, H.: Functional Analysis, Sobolev Spaces and Partial Differential Equations. Springer, New York (2011)

[9] Boudin, L., Grandmont, C., Moussa, A.: Global existence of solutions to the incompressible Navier-Stokes-Vlasov equations in a time-dependent domain. J. Differ. Equations 262(3), 1317-1340 (2017)

[10] Brown, R.M., Hu, W., Lieberman, G.M.: Weak solutions of parabolic equations in non-cylindrical domains. Proc. Am. Math. Soc. 125(6), 1785-1792 (1997)

[11] Byun, S.-S., Wang, L.: Parabolic equations in time dependent Reifenberg domains. Adv. Math. 212(2), 797-818 (2007)

[12] Calvo, J., Novaga, M., Orlandi, G.: Parabolic equations in time dependent domains. J. Evol. Equations 17(2), 781-804 (2017)

[13] Daners, D., Thornett, C.: Periodic-parabolic eigenvalue problems with a large parameter and degeneration. J. Differ. Equations 261(1), 273-295 (2016)

[14] Du, Y., Peng, R.: The periodic logistic equation with spatial and temporal degeneracies. Trans. Am. Math. Soc. 364(11), 6039-6070 (2012)

[15] Evans, L.C.: Partial Differential Equations. Graduate Studies in Mathematics, vol. 19. American Mathematical Society, Providence, RI (1998)

[16] Hess, P.: Periodic-Parabolic Boundary Value Problems and Positivity, Pitman Res., Notes in Mathematics, vol. 247. Longman Sci. Tech., Harlow (1991)

[17] Ladyzhenskaya, O.A., Ural'tseva, N.N.: Linear and Quasilinear Elliptic Equations. Academic Press, New York, London (1968)

[18] López-Gómez, J.: Protection zones in periodic-parabolic problems. Adv. Nonlinear Stud. 20, 253-276 (2020)

[19] Savaré, G.: Parabolic problems with mixed variable lateral conditions: an abstract approach. J. Math. Pures Appl. 76(4), 321-351 (1997) 
[20] Simon, B.: Semiclassical analysis of low lying eigenvalues. II. Tunneling. Ann. Math. 120(1), 89-118 (1984)

P. Álvarez-Caudevilla, F. Belinchón and C. Brändle

Universidad Carlos III de Madrid

Av. Universidad 30

28911 Leganés

Spain

e-mail: pacaudev@math.uc3m.es

F. Belinchón

e-mail: fbelinch@math.uc3m.es

C. Brändle

e-mail: cbrandle@math.uc3m.es

Received: October 23, 2020.

Revised: March 10, 2021.

Accepted: October 5, 2021. 